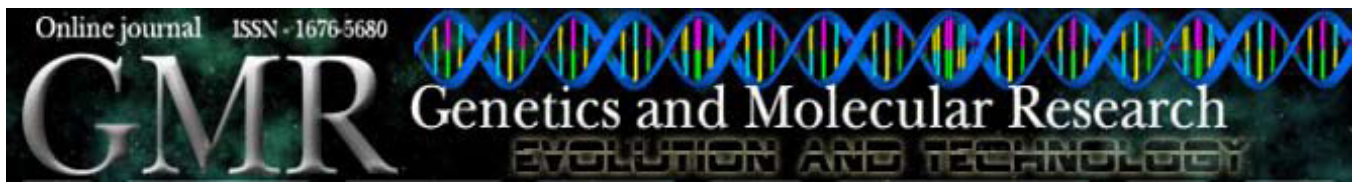

\title{
Identifying the molecular basis of functions in the transcriptome of the social amoeba Dictyostelium discoideum
}

\author{
T.J. Whitney ${ }^{1,2 *}$, D.G. Gardner ${ }^{1 *}$, M.L. Mott $^{1,3}$ and M. Brandon ${ }^{1,4}$ \\ ${ }^{1}$ Department of Biological Sciences, \\ Idaho State University, Pocatello, ID, USA \\ ${ }^{2}$ Current address: Department of Pathology, \\ University of Utah, Salt Lake City, UT, USA \\ ${ }^{3}$ Current address: Pritzker School of Medicine, \\ University of Chicago, Chicago, IL, USA \\ ${ }^{4}$ Current address: Fort Lewis College, \\ School of Natural and Behavioral Sciences, Durango, CO, USA \\ *These authors contributed equally to this study. \\ Corresponding author: M. Brandon \\ E-mail: Brandon_m@fortlewis.edu
}

Genet. Mol. Res. 9 (1): 394-415 (2010)

Received November 27, 2009

Accepted December 16, 2009

Published March 9, 2010

\begin{abstract}
The unusual life cycle of Dictyostelium discoideum, in which an extra-cellular stressor such as starvation induces the development of a multicellular fruiting body consisting of stalk cells and spores from a culture of identical amoebae, provides an excellent model for investigating the molecular control of differentiation and the transition from single- to multi-cellular life, a key transition in development. We utilized serial analysis of gene expression (SAGE), a molecular method that is unbiased by dependence on previously identified genes, to obtain a transcriptome from a high-density culture of amoebae, in order to examine the transition to multi-cellular development. The SAGE method pro-
\end{abstract}


vides relative expression levels, which allows us to rank order the expressed genes. We found that a large number of ribosomal proteins were expressed at high levels, while various components of the proteosome were expressed at low levels. The only identifiable transmembrane signaling system components expressed in amoebae are related to quorum sensing, and their expression levels were relatively low. The most highly expressed gene in the amoeba transcriptome, dutA untranslated RNA, is a molecule with unknown function that may serve as an inhibitor of translation. These results suggest that high-density amoebae have not initiated development, and they also suggest a mechanism by which the transition into the development program is controlled.

Key words: Dictyostelium discoideum; Amoebae; Transcriptome; SAGE

\section{INTRODUCTION}

The social amoeba Dictyostelium discoideum has long been a productive model for basic eukaryotic cell processes, including cytoskeletal structure and motility, transmembrane signaling, and regulation of gene expression (Chung and Firtel, 2000). In addition, this organism's unusual life cycle, in which an extra-cellular stressor like starvation induces the development of a multicellular fruiting body consisting of stalk cells and spores from a culture of identical amoebae, provides an excellent model for investigating the molecular control of differentiation and the transition from single cell to multi-cellular life, a key transition in development (Firtel, 1996; Parent and Devreotes, 1996; Verkerke-van Wijk et al., 2001).

The advent of microarray technology has provided opportunities to efficiently compare the expression of known genes between various stages of development. For example, Urushihara et al. (2004) conducted a large-scale comparison of Dictyostelium cDNAs from the amoeba and slug stages of the life cycle to look for differences in gene expression patterns. Van Driessche et al. (2002) examined the expression patterns of genes across the life cycle to predict those whose expressions change during development, and therefore are expected to contribute to morphological changes.

Publication of the Dictyostelium genome sequence (Eichinger et al., 2005) made it possible to utilize molecular methods that are not biased by a dependence on previously identified genes. One of these methods is serial analysis of gene expression (SAGE), a technique that produces a library of cDNA tags from total RNA isolated from cells at a particular stage of the life cycle. After sequencing, the tags can be rank ordered relative to the expression levels of all other tags in the library (Velculescu et al., 1995). Comparison of the tag sequences to the Dictyostelium genome sequence identifies the genes, and produces a transcriptome that describes, in rank order, the activities of the cells that served as the source of RNA.

We report the production of an SAGE library and transcriptome from a high-density culture of Dictyostelium amoebae. We analyzed the transcriptome to predict the functional capabilities of amoebae and their ability to transition to multicellular development. 


\section{MATERIAL AND METHODS}

\section{Cell maintenance and preparation}

We grew Dictyostelium discoideum AX2 wild-type cells in HL5 liquid media (0.06 M dextrose, $10 \mathrm{~g} / \mathrm{L}$ proteose peptone, $5 \mathrm{~g} / \mathrm{L}$ yeast extract, $0.004 \mathrm{M} \mathrm{Na}_{2} \mathrm{HPO}_{4}, 0.004 \mathrm{M} \mathrm{KH}_{2} \mathrm{PO}_{4}$ ), shaking at $21{ }^{\circ} \mathrm{C}$, until they reached a concentration of $2.4 \times 10^{7}$ cells $/ \mathrm{mL}$. We washed cells twice in starvation buffer $\left(100 \mathrm{~mL} 5 \mathrm{mM} \mathrm{Na}_{2} \mathrm{HPO}_{4}, 5 \mathrm{mM} \mathrm{NaH}_{2} \mathrm{PO}_{4}, 0.02 \mathrm{mM} \mathrm{CaCl}, 2 \mathrm{mM}\right.$ $\mathrm{MgSO}_{4}$ ), and finally resuspended the cells in $1 \mathrm{~mL}$ starvation buffer.

\section{RNA isolation}

We extracted total RNA from amoebae prepared as described above using Ambion's RNAqueous RNA Isolation Kit (Austin, TX, USA). In order to test the integrity of the RNA, we separated $2 \mu \mathrm{L}$ of each sample on a $1 \%(\mathrm{w} / \mathrm{v})$ agarose gel. Visualization of distinct $18 \mathrm{~S}$ and $28 \mathrm{~S}$ ribosomal RNA bands indicated that the RNA was intact. We measured the concentration and purity of the RNA using a Pharmacia Biotech Gene Quant II spectrophotometer, and used $8.7 \mu \mathrm{g}$ of total RNA as a starting point for SAGE.

\section{Serial analysis of gene expression}

We obtained an I-SAGE Kit from Invitrogen (San Diego, CA, USA) and followed the protocol and recommendations contained in the I-SAGE manual, with the modifications described below. In brief, we synthesized cDNA from the amoebae mRNA; then, through a series of restriction digests, we produced a 14-bp tag that was unique to each expressed gene. We ligated the tags into ditags, then into concatemers, and cloned and sequenced 30,830 tags.

In order to optimize our results, we modified several of the steps of the SAGE procedure. At various points in the SAGE procedure, wash steps, generally followed by brief (2-3 s) centrifugation, are recommended in order to recover all of the cDNA bound to magnetic beads. However, during cDNA synthesis we observed significant clumping of the beads after centrifugation, which severely limited cDNA recovery. Therefore, we eliminated the centrifugation steps. The final step in the preparation of $26 \mathrm{bp}$ ditags is ethanol precipitation and air-drying the DNA pellet. We replaced the air-drying step with removal of ethanol by pipetting and blotting the tubes on Kim-wipes as air-drying causes degradation of the $26 \mathrm{bp}$ ditags (Kenzelmann and Muhlemann, 1999).

We found that high ditag DNA concentrations inhibit ligation of ditags into concatemers. In order to maximize ligation of the $26 \mathrm{bp}$ ditags, we set up four different ligation reactions using $25 \%$ of the DNA in each tube, maintaining the manufacturer's suggested volumes of the other enzymes and reagents. Additionally, we added a second aliquot of T4 DNA ligase after $3 \mathrm{~h}$ of ligation, and increased the total ligation reaction time to $15 \mathrm{~h}$. Finally, we vortexed and briefly $(5 \mathrm{~s})$ microfuged the ligation reaction tubes periodically during the ligation reaction.

Prior to gel purification of the concatemers, we heated the concatemer ligation reaction mixture to $65^{\circ} \mathrm{C}$ for $20 \mathrm{~min}$ to release the partially hybridized concatemers. Finally, we lengthened the reaction time for ligation of concatemers into $\mathrm{pZErO}{ }^{\circledR}$ from 1 to $6 \mathrm{~h}$ at $16^{\circ} \mathrm{C}$. 


\section{Data analysis}

We used polymerase chain reaction (PCR) with M13 forward and reverse primers to amplify the cloned concatemers. We purified the PCR products using $40 \%(\mathrm{w} / \mathrm{v})$ polyethylene glycol, and submitted them for sequencing to the Idaho State University Molecular Research Core Facility, which employs an Applied Biosystems 3100 Genetic Analyzer (Foster City, CA, USA).

We entered the sequence files into the SAGE software that is included in the ISAGE kit. This software extracts individual tag sequences from the concatemer sequences, and compiles a list of each independent tag, and the number of times that tag is represented in the library. In order to identify the gene represented by each tag, we compared the specific tag sequence to the $D$. discoideum genome database (Chisholm et al., 2006) as well as the GenBank database, using BLAST (Basic Local Alignment Search Tool). All SAGE sequence tags were converted into FASTA format and a batch BLAST search was run against mRNA sequences downloaded from Genbank. Additionally, the SAGE sequence tags, in FASTA format, were individually imported into the query field of the nucleotide BLAST program located at www.ncbi.nlm.nih.gov. A mega BLAST search was then performed on the SAGE sequence tags using a $D$. discoideum (taxid:44689) curated, refseq mRNA, database. The BLAST program automatically adjusted the search parameters to search for a short input sequence. The best $D$. discoideum mRNA sequence alignment for each individual SAGE tag was then chosen only from those responses that had $100 \%$ alignment with the search tag. Due to the small size of the search tag, $14 \mathrm{bp}$, the e-value, or statistically significant value, was used only when two or more mRNA's had $100 \%$ alignment with the search tag; the mRNA with the lowest e-value was selected.

\section{RESULTS}

\section{General properties of the SAGE library}

The amoeba-stage SAGE library is based on the sequences of 30,830 individual tags. Of these, 27,412 tags appeared in the library in only one copy. Because we cannot distinguish between true extremely low copy number tags and sequencing errors, we did not report the single copy tags in the library. We discontinued tag sequencing at this point for several reasons. First, no tags shifted from the single copy category into a higher expression level category. Second, the relative expression levels of the most highly expressed genes (i.e., those expressed at the level of at least 13 copies) did not change with the identification of additional tags, suggesting that the library accurately represents the relationships between the expressed genes. These data suggest that amoebae express about 3418 genes at the level of at least two RNA molecules. Since the Dictyostelium genome predicts about 12,500 genes, the SAGE library predicts that about $27 \%$ of the genome is required for amoeba structure and functions (Eichinger et al., 2005). The complete list of SAGE tags and their relative expression levels is available at SAGEmap (accession number GSE12095). The amoeba-stage transcriptome described here consists of the 316 most highly expressed genes, that is, all genes expressed at the level of at least 13 copies (Table 1). 


\begin{tabular}{|c|c|c|c|}
\hline Dictyostelium tag sequence & ID & Count & Description \\
\hline GGACTTAGAG & EAL64828 & 773 & dutA untranslated RNA \\
\hline ATTAGAGGAC & $\begin{array}{l}\text { XM_629433.1, } \\
\text { XM_629434.1, } \\
\text { XM_635973.1, } \\
\text { XM_635972.1, } \\
\text { XM_635971.1 }\end{array}$ & 440 & Ubiquitin ( $u b q N, u b q O, u b q M, u b q L, u b q K)$ \\
\hline TGCAGACGTT & XM_640038.1 & 308 & 40S ribosomal protein S29 (rps29) \\
\hline CAAAGACTTT & XM_640564.1 & 297 & $\begin{array}{l}\text { AX4 putative glycoside hydrolase/ } \\
\text { Mus musculus zinc finger protein } 119 \text { (Zfp119) }\end{array}$ \\
\hline ATTTTTATTA & XM_633616.1 & 258 & $\begin{array}{l}\text { CHD gene family protein containing chromodomain, } \\
\text { helicase domain, and DNA-binding domain }\end{array}$ \\
\hline GGATAACAAA & EAL69803 & 256 & $\mathrm{G}$ beta-like protein $(\mathrm{g} p b B)$ \\
\hline GGGTATGGAT & XM_636628.2 & 195 & cysteine proteinase $(C P 4)$ \\
\hline TTTGGTTGGT & XM_632956.1 & 175 & calcium-dependent cell adhesion molecule-1 ( $\operatorname{cad} A)$ \\
\hline CTGGTTACTC & XM̄ō64088.1 & 168 & protein synthesis elongation factor 1 -alpha (tef2) \\
\hline GAAAGACGTC & XM_15384.1 & 168 & V12 mRNA/ribosomal protein 1024 (rps 9$)$ \\
\hline GAAAAGAGAA & XM_635906.1 & 155 & $40 \mathrm{~S}$ ribosomal protein $\mathrm{S} 6(r p s 6)$ \\
\hline CTCGTCCTTA & XM_636364.1 & 154 & ribosomal protein L18 (rpl18) \\
\hline TCGCTTTAAT & XM_634269.1 & 154 & hypothetical protein \\
\hline GATATTGAAG & XM_637875.1 & 151 & ribosomal protein L10a $(r p l 10 \mathrm{a})$ \\
\hline TGGTTATCCA & XM_637389.2 & 142 & $\begin{array}{l}\text { Dictyostelium discoideum } \mathrm{AX} 4 \text { putative transport protein } \\
\text { (sec241) mRNA, complete cds }\end{array}$ \\
\hline GCAATCAAAA & XM_630714.1 & 140 & hypothetical protein \\
\hline GGGTATCGAA & XM_639885.1 & 140 & cysteine proteinase CP5 (cprE) \\
\hline TGTAGAGGTG & XM_637392.1 & 139 & 60S ribosomal protein L4 (rpl4) \\
\hline CTACTTTCGA & XM_635251.1, U13671.1 & 138 & Actin binding protein (hat $B) /$ hisactophilin II $($ hsII) \\
\hline CAAGTTGAAC & AB000109.1 & 133 & mitochondrial DNA \\
\hline GTATAGCTTA & EAL64828 & 130 & dutA RNA \\
\hline CGTAAACCAG & X15383.1 & 122 & Ribosomal protein ( $r p 119)$ vegetative specific V14 gene \\
\hline GTGGTTTGAA & XM_639670.1 & 112 & $40 \mathrm{~S}$ ribosomal protein $\mathrm{S} 30$ (rps 30$)$ \\
\hline CACTAATCAA & XM_635539.1 & 111 & hypothetical protein \\
\hline GGGTAAGATT & XM_641702.1 & 110 & ribosomal protein $\mathrm{L} 35 \mathrm{a}(r p l 35 \mathrm{a})$ \\
\hline TGAAATATAT & XM_001732911.1 & 104 & putative polyketide synthase $(p k s)$ \\
\hline GGGTGATAAT & XM_633636.1 & 103 & histidine kinase (dhkG) mRNA, complete cds \\
\hline GCCACTTTCT & XM_628965.1 & 100 & ribosomal protein s2 (rps2)/LLRep3 \\
\hline GGGTGCCGAT & XM_630282.1 & 95 & hypothetical protein \\
\hline TGCTATCCAC & XM_635481.1 & 95 & $40 \mathrm{~S}$ ribosomal protein S26 (rps 26$)$ \\
\hline AAAATTAAAA & XM_641596.1 & 94 & $\operatorname{actin}(a c t 8)$ \\
\hline AAAATTAAAA & XM_640170.1 & 94 & actin $16($ act 16$)$ \\
\hline GCCGCTCAAG & XM_630185.1 & 94 & 40 S ribosomal protein $\mathrm{S} 14$ (rps 14$)$ \\
\hline TGGTTACAAG & XM_641274.1 & 94 & ribosomal protein $\mathrm{L} 36 \mathrm{a}(\mathrm{rpl36 \textrm {a }})$ \\
\hline AGAGGTCTCA & XM_636637.1 & 93 & $\begin{array}{l}\text { vegetative specific gene V18 gene for ribosomal protein/ } \\
\text { ribosomal protein L11 ( } r p l 11)\end{array}$ \\
\hline GTCGTCCAGG & XM_633034.1 & 92 & $40 \mathrm{~S}$ ribosomal protein $\mathrm{S} 15(\operatorname{rps} 15)$ \\
\hline TAACAACCGT & XM_641608.1 & 90 & $40 \mathrm{~S}$ ribosomal protein $\mathrm{SA}(\operatorname{rps} A)$ \\
\hline GTCGTTCTTA & X00 0134.1 & 88 & $17 \mathrm{~S}$ small subunit ribosomal RNA gene \\
\hline GTGTTAACGA & XM_639972.1 & 84 & ribosomal protein L15 (rpl15) \\
\hline GGTACCAAAG & $\begin{array}{l}\text { XM_636854.1, } \\
\text { XM_642089.1 }\end{array}$ & 82 & ribosomal protein L31 ( $r p l 31) /$ putative myb transcription factor \\
\hline CCACTACAAC & $\begin{array}{c}\text { X'- } 15710.1 \\
\text { XM_639100.1 }\end{array}$ & 80 & ribosomal protein L2/60S ribosomal protein L8 ( $r p l 8$ ) \\
\hline TGAAATAAAA & XM_637148.1 & 79 & extracellular matrix protein ST430 (13/13) \\
\hline TGCTGTCTCT & XM_633409.1 & 79 & glycyl-tRNA synthetase $($ glys $)(12 / 12)$ \\
\hline GTGTAATCCA & EĀL63602 & 77 & small aggregate formation $(\mathrm{smlA})$ \\
\hline TTTAAGTGGT & XM_642699.1 & 76 & P17 protein \\
\hline AAATATAAAA & XM_635590.1 & 72 & hypothetical protein \\
\hline CGCTGGTGCA & XM_631726.1 & 69 & ribosomal protein L10E $(r p l 10)$ \\
\hline ATCAAATCTG & $\begin{array}{l}\mathrm{XM} 629345.1 \\
\mathrm{U} 78756.1\end{array}$ & 68 & $40 \mathrm{~S}$ ribosomal protein $\mathrm{s} 3(r p s 3),(r p g G)$ \\
\hline
\end{tabular}

Continued on next page 
Table 1. Continued.

\begin{tabular}{|c|c|c|c|}
\hline Dictyostelium tag sequence & ID & Count & Description \\
\hline GAGAATCATC & XM_632378.1 & 68 & hypothetical protein \\
\hline GAAGTCGGTA & AY171066.1 & 64 & extrachromosomal palindromic ribosomal RNA \\
\hline GATGTCATCT & XM_639821.1 & 64 & 40S ribosomal protein $\mathrm{S} 4$ (RPS4) \\
\hline CCTCTGGTAT & XM_640073.1 & 63 & $40 \mathrm{~S}$ ribosomal protein $\mathrm{S} 23$ ( $r p s 23$ ) \\
\hline GAGAAAACCA & XM_640259.1 & 63 & ribosomal protein L32 (rpl32) \\
\hline AAAAATCAAA & XM_639860.2 & 62 & putative polyketide synthase ( $p k s 15$ ) \\
\hline GATAAAAAAG & XM_637269.1 & 62 & $40 \mathrm{~S}$ ribosomal protein $\mathrm{S} 20$ (rps 20$)$ \\
\hline GTAAACCAAT & XM_638427.1 & 61 & AX4 RNA polymerase I, largest subunit (rpa1) \\
\hline CCAAAAGATA & XM_642485.1 & 59 & histone $\mathrm{H} 3(d d H 3)$ \\
\hline GAAAAAGGTT & AF095929.1 & 59 & actin related protein $2(\operatorname{arp} B)$ \\
\hline GGTAATGGTA & XM_634924.1 & 59 & cyclophilin $(p p i A)$ \\
\hline TCCCTATTAA & XM_632008.1 & 59 & hypothetical protein \\
\hline GCTGCTCACT & XM_636632.1 & 57 & ribosomal protein L21 (rpl21) \\
\hline GGGTGCTGAC & XM_631551.1 & 57 & hypothetical protein \\
\hline GTTGTAACGG & X0̄0134.1 & 54 & 18S rRNA gene \\
\hline ATGAAAGTGC & M25217.1 & 53 & $\operatorname{actin}(a c t)$ \\
\hline AATCACCCAA & XM_638028.1 & 52 & 40S ribosomal protein $\mathrm{S} 18$ (rps 18$)$ \\
\hline GGCCACCGAA & XM_629870.1 & 52 & $40 \mathrm{~S}$ ribosomal protein $\mathrm{S} 8$ ( $r p s 8)$ \\
\hline TAAGATTGTT & X1̄14970.1 & 52 & translation initiation factor $e I F-4 D$ \\
\hline CTTGTGAGTT & XM_642699.1 & 51 & 17-kDa protein (p17) \\
\hline TTCAACTGTT & XM_637485.1 & 51 & $40 \mathrm{~S}$ ribosomal protein $\mathrm{S} 27$ (rps27) \\
\hline GTATGATAAT & XM_638765.1 & 50 & glyceraldehyde-3-phosphate dehydrogenase ( $g p d A$ ) \\
\hline GTGAATCAAA & XM_635424.1 & 49 & putative alpha-amylase $($ amy $A)$ \\
\hline TGTCGATCCA & XM_641310.1 & 49 & hypothetical protein \\
\hline TGCTACTATC & XM_642726.1 & 48 & 60 S ribosomal protein L9 $(r p l 9)$ \\
\hline TGGTGATGGT & XM_635474.1 & 46 & PhoPQ-activated pathogenicity-related protein $($ apr $A)$ \\
\hline AAAGAAGATG & XM_642543.1 & 45 & S-adenosyl-L-homocysteine hydrolase $(\operatorname{sah} A)$ \\
\hline GCAGGAAAAA & XM_636572.1 & 45 & hypothetical czl protein \\
\hline AAAAATATAG & $\mathrm{AB} 000109.1$ & 44 & mitochondrial DNA \\
\hline CAGTAGCTAA & AY007805.1 & 44 & ribosomal protein L36 \\
\hline CAGTTGATCC & XM_641577.1 & 44 & gelation factor $(a b p c C)$ \\
\hline CGAATGGAAT & XM_642251.1 & 44 & actin binding protein (coronin) ( $\operatorname{cor} A)$ \\
\hline AATTATTGAA & AF309947.1 & 43 & $\operatorname{Rac1A}(\operatorname{raclA})$ \\
\hline AATTCTTAAT & $\begin{array}{l}\text { XM_629919.1, } \\
\text { XM_629706.1 }\end{array}$ & 43 & ribosomal protein (L3) gene/AX4 aminomethyltransferase $(r p l 3)$ \\
\hline CTGATAATGA & $\begin{array}{c}\mathrm{XM}_{\mathrm{U}}^{-} 642071.1 \\
\mathrm{U} 23957.1\end{array}$ & 42 & acetylornithine deacetylase $(\arg E) / \mathrm{P} 52 \mathrm{D}$ \\
\hline TACTCTTTCT & AF203735.1 & 42 & culmination specific protein 45D (45D) \\
\hline TTGACATTGA & M91383.1 & 42 & thioredoxin $(\operatorname{tr} x 3)$ \\
\hline AACTCTGGTC & XM_632836.1 & 41 & 40S ribosomal protein $\mathrm{s} 5$ (rps5) \\
\hline GAACCAGCCT & XM_638827.1 & 41 & hypothetical protein \\
\hline GGATCCAGTG & XM_641936.1 & 41 & hypothetical protein DDB_0190094 partial mRNA \\
\hline AGTGTCGCTG & XM_631261.1 & 39 & ADP-ribosylation factor 1 \\
\hline CCAACCCGTT & XM_640574.1 & 39 & ribosomal protein L27 (rpl27) \\
\hline GGGTAATAAT & XM_629547.1 & 39 & discoidin II $(d s c E)$ \\
\hline GTTATTGTAG & XM_638733.1 & 39 & conditioned medium factor $(\mathrm{cmfA})$ \\
\hline GTTGGGAAGA & AY0440851.1 & 39 & Rho GDP-dissociation inhibitor (rdiA) \\
\hline CAAAACCCAG & U78759.1 & 38 & IfdA translation initiation factor (ifdA) \\
\hline GAAAGATGGA & XM_639860 & 38 & putative polyketide synthase ( $p k s 15)$ \\
\hline TCCATCATCA & XM_640805.1 & 37 & EGF-like domain \\
\hline TCCGCCTTAG & XM_632431.1 & 37 & $\begin{array}{l}\text { putative GATA-binding transcription factor } \\
\text { (DDB_0216328) mRNA, complete cds }\end{array}$ \\
\hline AAAATAATAA & XM_636161.1 & 36 & YELA translation initiation factor (yelA) \\
\hline AAAATTTACA & XM_001732912.1 & 36 & putative polyketide synthase \\
\hline AAAGTTAGAG & XM̄_629874.1 & 36 & ribosomal protein L13 (rpl13) \\
\hline GCATTTGGAA & XM_631261.1 & 36 & ADP-ribosylation factor $(\operatorname{arf} A)$ \\
\hline TGTAACAACA & XM_632548.1 & 36 & putative actin binding protein \\
\hline GTGTGGTGAA & $\begin{array}{l}\text { M9-2992.1, } \\
\text { XM_628883.1 }\end{array}$ & 35 & cyclic AMP-regulated protein ( $\mathrm{p} 16)$ /actin binding protein \\
\hline
\end{tabular}

Continued on next page 
Table 1. Continued.

\begin{tabular}{|c|c|c|c|}
\hline Dictyostelium tag sequence & ID & Count & Description \\
\hline TAAGAGTTTT & XM_631880.1 & 35 & $26 \mathrm{~S}$ proteasome regulatory subunit $\mathrm{S} 1$ ( $\mathrm{psmDl}$ ) \\
\hline AGTTTCAGCC & XM_640335.2 & 34 & hypothetical protein \\
\hline CCATCTTACA & XM_632592.1 & 34 & histone $\mathrm{H} 2 \mathrm{~B}$ domain containing protein $(\mathrm{H} 2 \mathrm{Bv} 3)$ \\
\hline GGGTCTTGAC & XM_636633.1 & 34 & cysteine proteinase $(c p r F) /$ cathepsin $\mathrm{O} ?$ \\
\hline TACTTTACCC & XM_639670.1 & 34 & 40S ribosomal protein $\mathrm{s} 30(\operatorname{rps} 30)$ \\
\hline GGAAATTTCT & XM_636553.1 & 33 & cathepsin $\mathrm{D}($ cts $D)$ \\
\hline GGGTACATAT & XM_636202.1 & 33 & hypothetical protein \\
\hline GGTATGATGT & XM_638942.1 & 33 & hypothetical protein \\
\hline AAGTCATCGA & XM_630012.1 & 32 & hypothetical protein $(29 \mathrm{C})$ \\
\hline ACGAACTTTA & XM_633918.1 & 32 & calreticlin $(\operatorname{crtA}) / \operatorname{grp} 94$ mRNA for glucose-regulated protein 94 \\
\hline AGAGGTATCC & XM_631629.1 & 32 & elongation factor 2 \\
\hline ATGAGCAGTT & EĀL63335 & 32 & mitochondrial DNA \\
\hline CACACCAGTA & XM_634256.1 & 32 & $\mathrm{p} 34-\operatorname{Arc}(\operatorname{arp} E)$ \\
\hline TAGATCAGAA & XM_634139.1 & 32 & phosphopyruvate hydratase (enoA) \\
\hline TCCCCGTACA & XM_633420.1 & 32 & NOT2/NOT3/NOT5 family protein \\
\hline TGTTCACCAT & XM_633215.1 & 32 & ribosomal protein L17 (spl17) \\
\hline GACCACAACG & XM_637999.1 & 31 & $40 \mathrm{~S}$ ribosomal protein $\mathrm{s} 15(\operatorname{rps} 15 \mathrm{a}) / 40 \mathrm{~S}$ ribosomal protein $\mathrm{S} 24$ \\
\hline TTTGTCAAGA & XM_631966.1 & 31 & alpha tubulin $(t u b a)$ \\
\hline CAAATGTAGT & XM_633133.1 & 30 & RING Zn finger-containing protein (DDB-0232321) \\
\hline TAAAAGCTCT & EĀL66985 & 30 & $\mathrm{p} 41-\operatorname{Arc}(\operatorname{arp} D)$ \\
\hline TTTGGTTATG & XM_630291.1 & 30 & S-adenosylmethionine synthetase (met $K$ ) \\
\hline AAGAACAAGC & XM_641070.1 & 29 & beta tubulin gene $(t u b B)$ \\
\hline CAATTCGATA & XM_641945.1 & 29 & vacuolar protein sorting-associated protein (vps26) \\
\hline GGATGATGAA & XM_6288̄65.1, U15926.1 & 29 & putative polyketide synthase/elongation factor $1 \mathrm{~b}(p k s 1)$ \\
\hline GGATGGTGGT & XM_638832.1 & 29 & acetyl-coA C-acyltransferase \\
\hline GTCATTTCTT & XM_629407.1 & 29 & $\begin{array}{l}\text { putative protein tyrosine phosphatase, dual specificity } \\
\text { (DDB_0238566) mRNA, complete cds }\end{array}$ \\
\hline TGGATGTGAC & XM_001732949.1 & 29 & putative polyketide synthase $(p k s)$ \\
\hline AGCTGGGTTT & EAL63335 & 28 & mitochondrial DNA \\
\hline TGTTGCCCTC & XM_637474.1 & 28 & $60 \mathrm{~S}$ ribosomal protein $\mathrm{L} 7 \mathrm{a}(\mathrm{rpl7a})$ \\
\hline AAGAAAACTC & XM_638053.1 & 27 & ribosomal protein L7 ( rpl7) \\
\hline AGATTCAAAT & XM_631705.1 & 27 & ribosomal protiein L12 (rpl12) \\
\hline ATATGACCCA & XM_632900.1 & 27 & $\begin{array}{l}\text { type I phosphodiesterase nucleotide phosphatase family protein/ } \\
\text { PkiA (pkiA) }\end{array}$ \\
\hline CTAATCGTCA & XM_637723.1 & 27 & ubiquitin $(u b q C)$ \\
\hline GATAAACAAA & XM_640344.1 & 27 & porin $($ por $A)$ \\
\hline TTGAAACTCA & XM_640947.1 & 27 & actinobindin $(a b n B)$ \\
\hline AAATGTGATG & $\begin{array}{l}\text { XM_641596.1, } \\
\text { XM_640170.1 }\end{array}$ & 26 & $\operatorname{actin}(\operatorname{act} 8) / \operatorname{actin}(\operatorname{act} 16)$ \\
\hline CAATGTTGAA & EĀL71934 & 26 & РерA \\
\hline CTTGGACTAA & XM_633462.1 & 26 & $40 \mathrm{~S}$ ribosomal protein $\mathrm{s} 13$ (rps 13$)$ \\
\hline AAGAAAGCTG & XM_632648.1 & 25 & myosin II heavy chain $(m h c A)$ \\
\hline GCGAATGCAC & XM_642513.1 & 25 & hypothetical protein \\
\hline TAAACGAATC & XM_638093.1 & 25 & cytoplasmic dynein heavy chain $(d h c A)$ \\
\hline TCTCAAGATC & AY007804.1 & 25 & ribosomal protein L28 (rpl28) 13/13 \\
\hline CTCGCTCAAA & XM_636770.1 & 24 & 30,000 dalton actin bundling protein $(a b p B)$ \\
\hline GAGATAATCT & XM_631114.1 & 24 & hypothetical protein \\
\hline GAGTAAAGAT & XM_639850.1 & 24 & $\operatorname{CsbA}(c s b A)$ and $\operatorname{CsbB}(c s b B)$ \\
\hline GGCTCTCTCT & XM_638782.1 & 24 & fructose-bisphosphate adolase $(f b a)$ \\
\hline GTTTGTTTGG & XM_640382.1 & 24 & EGF-like domain containing protein ( $\mathrm{canC}$ ) \\
\hline TAATAAACTC & AC116986.1 & 24 & chromosome 2 map \\
\hline AAATCAAAAA & XM_001732910.1 & 23 & putative polyketide synthase \\
\hline CATTCGTAAA & X⿳亠丷⿵冂_640374.1 & 23 & proteosomal alpha-subunit 7-1 (pntB) \\
\hline GCTACCGTCA & XM_630705.1 & 23 & ribosomal protein L23 (rpl23) \\
\hline GTTTCTAAAA & XM_641945.1 & 23 & vacuolar protein sorting-associated protein \\
\hline GTTTTGGTGG & XM_640757.1 & 23 & hypothetical protein \\
\hline TAAATAGATC & XM_630528.1 & 23 & hypothetical protein \\
\hline TCATTCGTTT & XM_638104.1 & 23 & glutamine amidotransferase (pyr1-3) \\
\hline TCATTCGTTT & XM_638093.1 & 23 & cytoplasmic dynein heavy chain $(d h c A)$ \\
\hline
\end{tabular}

Continued on next page 
Table 1. Continued.

\begin{tabular}{|c|c|c|c|}
\hline Dictyostelium tag sequence & ID & Count & Description \\
\hline AAATCGCTTT & XM_636325.1 & 22 & nucleoside diphosphate kinase $(n d k M)$ \\
\hline AAGATGATGA & XM_638093.1 & 22 & heat shock protein \\
\hline CAGCACTAAA & M91382.1 & 22 & thioredoxin $(\operatorname{tr} x 2)$ \\
\hline CGAATTTATG & XM_640151.1 & 22 & actin capping protein CAP34 protein \\
\hline GATTATCTTA & XM_632335.1 & 22 & polyketide synthase ( $p k s 25)$ \\
\hline GCTTTGGATG & XM_632592.1 & 22 & histone $\mathrm{H} 2 \mathrm{~B}$ domain containing protein $(\mathrm{H} 2 \mathrm{Bv} 3)$ \\
\hline GTCACTTCTT & XM_639759.1 & 22 & hypothetical protein \\
\hline GTGAAGATAA & XM_641945.1 & 22 & $\begin{array}{l}\text { vacuolar protein sorting-associated protein (DDB_0234203) } \\
\text { mRNA, complete cds }\end{array}$ \\
\hline AATGCTTTCA & XM_632600.1 & 21 & $60 \mathrm{~S}$ ribosomal acidic phosphoprotein $\mathrm{P} 0$ \\
\hline ATCATCAATA & XM_637516.1 & 21 & vacuolar $\mathrm{H}+\mathrm{ATPase} \mathrm{B}$ subunit (vatB) \\
\hline CGCAAATGGT & XM_633239.1 & 21 & NADH dehydrogenase (ubiquinone) (DDB_0233208) \\
\hline CTGATCAAGA & XM_642037.1 & 21 & superoxide dismutase $(\operatorname{sod} A)$ \\
\hline GCTGTCGTCA & EĀL63335 & 21 & mitochondrial DNA \\
\hline GGATCTGCTG & XM_630177.1 & 21 & hypothetical protein \\
\hline GTCAAGCAAA & XM_632222.2 & 21 & hypothetical protein \\
\hline GTGGTAGTAG & XM_642014.1 & 21 & diaminopimelate epimerase (dafE) \\
\hline GTTAGAGCCA & XM_641467.1 & 21 & succinate dehydrogenase (ubiquinone) \\
\hline TAAAAAAAAA & $\mathrm{AC} 117072.3$ & 21 & chromosome 2 map \\
\hline TAAATTGGAC & XM_637030.1 & 21 & 6-phosphogluconate dehydrogenase (gnd) \\
\hline TAGAAGAAGA & XM_642384.1 & 21 & non-transporter $\mathrm{ABC}$ protein $(a b c F 4)$ \\
\hline TATTGGTCAT & EĀL63838 & 21 & V-ATPase A subunit (vatA) \\
\hline TGCTCTGTAG & XM_639875.1 & 21 & major facilitator superfamily protein DDB_0238776 \\
\hline TGCTCTGTAG & XM_631290.1 & 21 & myosin IB $(m y o B)$ mRNA \\
\hline TTGCAGAAAT & XM_630145.1 & 21 & hypothetical protein DDBdraft_0183916 \\
\hline AATTTGATGT & XM_639860.2 & 20 & putative poyketide synthase $(p \bar{k} s 15)$ \\
\hline CTAATGGGTC & XM_638179.1 & 20 & hypothetical protein (DDB_0232153) \\
\hline GCTAGAAATG & XM_632378.1 & 20 & hypothetical protein (DDBdraft_0187197) \\
\hline GTAATATTGT & XM_640523.2 & 20 & BEACH domain containing protein $(l v s B)$ \\
\hline GTCTTCAAGA & XM_639159.1 & 20 & colossin B mRNA ( $\operatorname{colB})$ \\
\hline TTATTGATTA & XM_641945.1 & 20 & vacuolar protein sorting-associated protein (DDB_0234203) \\
\hline TTTTCAATAA & XM_639838.2 & 20 & putative polyketide synthase ( $p k s 14)$ \\
\hline AAAAACACAT & XM_642213.1 & 19 & hyothetical protein DDBdraft_0216555 \\
\hline AAAAACACAT & XM_641971.1 & 19 & hypothetical protein DDBdraft_ 0216640 \\
\hline AAAAACACAT & XM_642177.1 & 19 & hypthetical protein DDBdraft_o 0216547 \\
\hline AAAAAGAAAT & EĀL68664 & 19 & clathrin light chain $($ clcA $)$ \\
\hline AGCAGAAGCA & XM_640867.2 & 19 & hypothetical protein DDBdraft_0215165 \\
\hline ATGCTCCAAT & XM_001733034.1 & 19 & hypothetical protein DDBdraft_0215064 \\
\hline ATGCTCCAAT & X⿳亠丷厂二_628847.1 & 19 & leucine-rich repeat-containing protein $(\operatorname{lr} r A)$ \\
\hline GGAATTACCA & XM_632762.1 & 19 & hypothetical protein DDBdraft_0215883 \\
\hline GTACTGAATT & EĀL64547 & 19 & crystal protein $($ cryS $)$ \\
\hline TAAAATTTTG & EAL71482 & 19 & rtoA \\
\hline TGGACCAAAG & XM_633814.1 & 19 & hypothetical protein DDB_0231746 \\
\hline CTCGTGTCTA & XM_629958.1 & 18 & cystatin A1 (cpiA) mRNA, complete cds \\
\hline CTGCTATTTC & XM_632737.1 & 18 & hypothetical protein DDBdraft_0186902 \\
\hline GAGAACTTAT & XM_632346.1 & 18 & SNF7 family protein ( $v p s 20)$ mRNA, complete cds \\
\hline GGTTGGTAGT & XM_631716.1 & 18 & leucine-rich repeat-containing protein $(\mathrm{lrr})$ ( $\mathrm{roco} 4)$ \\
\hline GTGATCTGTA & XM_637067.1 & 18 & hypothetical protein DDBdraft_0204455 \\
\hline GTGATCTGTA & XM_635945.1 & 18 & hypothetical protein DDBdraft_0204740 \\
\hline GTGGTGGGAA & XM_640047.1 & 18 & $\begin{array}{l}\text { putative small nuclear ribonucleoparticle-associated protein } \\
\text { DDB_0233178 }\end{array}$ \\
\hline GTTGGTACTC & XM_631286.1 & 18 & ankyrin repeat-containing protein DDB_0232969 \\
\hline TAAAACAATT & XM_639838.2 & 18 & putative polyketide synthase $(p k s 15)$ \\
\hline TACCAAAATG & XM_640812.1 & 18 & hypothetical protein DDBdraft_0201696 \\
\hline TGCTAACCTT & XM_632744.1 & 18 & alkyl-dihydroxyacetonephosphate synthase (agps) \\
\hline TGCTAACCTT & XM_636773.1 & 18 & hypothetical protein DDBdraft_0205035 \\
\hline TTGCTTCTGA & XM_639860.2 & 18 & putative polyketide synthase $(\overline{p k} s 15)$ \\
\hline AAGACACCAG & XM_639730.1 & 17 & heat shock protein $(h s p E-1)$ mRNA, complete cds \\
\hline AAGACACCAG & XM_639336.1 & 17 & heat shock protein (hspE-2) mRNA, complete cds \\
\hline
\end{tabular}

Continued on next page 
Table 1. Continued.

\begin{tabular}{|c|c|c|c|}
\hline Dictyostelium tag sequence & ID & Count & Description \\
\hline AAGATTAAAG & XM_641717.1 & 17 & $\begin{array}{l}\text { putative protein serine/threonine kinase DDB_0230126 } \\
\text { partial mRNA }\end{array}$ \\
\hline CACAAGAAAC & XM_633628.1 & 17 & P-type ATPase (atp $7 \mathrm{a})$ \\
\hline CACACCAACA & XM_- 638837.1 & 17 & hypothetical protein DDBdraft 0217482 \\
\hline GGAAACCTTT & XM_-639002.1 & 17 & hypothetical protein DDBdraft_0217527 \\
\hline GGAGGTATGC & XM_641119.1 & 17 & WH2 domain-containing protein DDB_0231673 \\
\hline GGTGCCATCG & XM_6-638855.1 & 17 & amidophosphoribosyltransferase $($ pur $\bar{F})$ \\
\hline GTAAATCATT & XM_001134471.1 & 17 & annexin VII $(n x n A)$ \\
\hline TAACAAAGAA & XM̄_640523.2 & 17 & $\begin{array}{l}\text { hypothetical protein BEACH domain-containing } \\
\text { protein }(l v s B) \text { mRNA }\end{array}$ \\
\hline TCAAATGCTG & XM_641218.1 & 17 & major vault protein alpha ( $m v p$-alpha) \\
\hline TGAAAAAAAA & AF4̄ 82390.1 & 17 & transporter AbcG11 $(a b c G 11)$ \\
\hline TGCAGTTTTA & XM_632827.2 & 17 & adenosine kinase $(a d k)$ \\
\hline TGGACTAAAT & XM_-638093.1 & 17 & hypothetical protein cytoplasmic dynein heavy chain $(d h c A)$ \\
\hline AAGCACAGGT & XM_630148.1 & 16 & $\begin{array}{l}\text { hypothetical protein RING Zn finger-containing protein (vps } 8) \\
\text { mRNA, complete }\end{array}$ \\
\hline AATGGATCAA & AY171066.1 & 16 & extrachromosomal palindromic ribosomal RNA \\
\hline ACTAATCAAT & XM_638814.1 & 16 & hypothetical protein \\
\hline ATCAATGGGT & XM_639599.1 & 16 & discoidin-I D chain $(d s c D)$ \\
\hline ATGATGATGA & XM_633148.1 & 16 & nucleotide binding protein 1 -like protein \\
\hline CAACCTCTGG & XM_629574.1 & 16 & hypothetical protein colossin A (colA) mRNA, complete cds \\
\hline CAACTCAATA & XM_001134515.1 & 16 & severin $(\operatorname{sev} A)$ \\
\hline CGATTCAATT & X⿳亠丷厂二 632550.1 & 16 & putative actin binding protein \\
\hline CGTAGAAAGC & XM_635431.1 & 16 & hypothetical protein \\
\hline CTTTCCGTGC & L46371 & 16 & cortexillin II \\
\hline GATGATGTAC & XM_636206.1 & 16 & hypothetical protein \\
\hline GTCTCTCCAG & XM_-632040.1 & 16 & RasGTPase-activating protein \\
\hline GTGAAAACAA & XM_635685.1 & 16 & hypothetical protein \\
\hline GTTCACTCCT & XM_633237.1 & 16 & fatty acid desaturase $(\mathrm{fad} A)$ \\
\hline TCAATTCGAT & XM_633521.1 & 16 & $\begin{array}{l}\text { ERG4/ERG24 ergosterol biosynthesis protein family } \\
\text { protein (DDB_0232079) }\end{array}$ \\
\hline TGAAGATGGT & XM_633121.1 & 16 & hypothetical protein \\
\hline TGGAAATGGT & XM_637070.1 & 16 & EGF-like domain-containing protein \\
\hline AAAACCATTG & XM_- 638265.1 & 15 & protein disulfide isomerase (dis) \\
\hline AAACCGTCAA & XM_633574.1 & 15 & $40 \mathrm{~S}$ ribosomal protein $\mathrm{S} 12$ ( $r p s 12)$ \\
\hline AAGAGTGGTA & XM_-641660.1 & 15 & ribosomal protein L30 (rpl30) \\
\hline ATAGAATGAG & XM_630305.1 & 15 & $\begin{array}{l}\text { penta EF hand calcium binding protein (pefA)/ } \\
\text { apoptosis-linked gene } 2(\operatorname{alg} 2 \mathrm{~A})\end{array}$ \\
\hline ATGATGAAGC & XM_629890.1 & 15 & vacuolar proton ATPase 100-kDa subunit (vatM) \\
\hline ATGTTTGGTG & XM_639226.1 & 15 & hypothetical protein \\
\hline ATTGAGAGAG & XM_-642726.1 & 15 & $60 \mathrm{~S}$ ribosomal protein L9 $(r p l 9)$ \\
\hline ATTGCTTCTT & XM_634949.1 & 15 & putative actin binding protein (DDB_0232396) \\
\hline CCGGATCACT & AY171065S2 & 15 & extrachromosomal palindromic ribosomal RNA \\
\hline GAAAATGGCC & XM_638890.1 & 15 & $\begin{array}{l}\text { hypothetical protein peptidase C19 family protein } \\
\text { (DDB_0237738) mRNA }\end{array}$ \\
\hline GAATTTTAAA & XM_639838.2 & 15 & $\begin{array}{l}\text { hypothetical protein putative polyketide synthase } \\
\text { (pks14) mRNA, complete cds }\end{array}$ \\
\hline GGAAAATACA & XM_641146.2 & 15 & hypothetical protein \\
\hline GTCACCACTA & XM_633438.1 & 15 & $\begin{array}{l}\text { pleckstrin homology }(\mathrm{PH}) \text { domain-containing protein }(\mathrm{gxc} Q) \\
\text { mRNA, complete cds }\end{array}$ \\
\hline GTGGTAGTTA & $\mathrm{AC} 114263$ & 15 & chromosome 2 map \\
\hline GTTGATGGGG & XM_641596.1, XM_640170.1 & 15 & $\operatorname{actin}(a c t 8) /$ actin $(a c t 16)$ \\
\hline TAAAAACAAC & XM_638317̄.1 & 15 & 13/13 natural resistance-associated macrophage protein ( \\
\hline TAAAGTTAGA & XM_632583.1 & 15 & $\begin{array}{l}\text { hypothetical protein vacuolar protein sorting-associated } \\
\text { protein mRNA }\end{array}$ \\
\hline TATCTCAAGA & XM_629630.1 & 15 & $\begin{array}{l}\text { 26S proteosome subunit ATPase } 5 \text { ( } p s m C 5) / \mathrm{HIV} 1 \text { TAT-binding } \\
\text { protein homologue }(t b p 1 \mathrm{O})\end{array}$ \\
\hline TATTTGATAC & XM_633831.1 & 15 & mMob1-like protein \\
\hline AGATCATCTC & XM_642439.1 & 14 & rab GTPase (rab6) \\
\hline
\end{tabular}

Continued on next page 
Table 1. Continued.

\begin{tabular}{|c|c|c|c|}
\hline Dictyostelium tag sequence & ID & Count & Description \\
\hline ATGATCGTGG & $\begin{array}{c}\text { XM_635251.1 } \\
\text { U13671.1 }\end{array}$ & 14 & actin binding protein $(h a t B) /$ hisactophilin II ( $h s I I)$ \\
\hline ATGCTGTCAT & XM_001134481.1 & 14 & $\begin{array}{l}\text { hypothetical protein centrosomal protein CP224 ( } m t a A) \\
\text { mRNA, complete cds }\end{array}$ \\
\hline CAAGTCGATA & XM_630022.1 & 14 & $\begin{array}{l}\text { GlcNAc transferase }(g n t 2) / \text { putative phosphatidylinositol } \\
\text { 4-phosphate 5-kinase }\end{array}$ \\
\hline CTGGTCTTGT & XM_631976.1 & 14 & hypothetical protein \\
\hline GAGAAGAAGA & XM_638794.1 & 14 & pleckstrin homology $(\mathrm{PH})$ domain-containing protein \\
\hline GATTGTCCAG & XM_635847.1 & 14 & ribonuclease $\mathrm{T} 2(\mathrm{ddiA})$ \\
\hline GCCGTGATGA & XM_632857.2 & 14 & hypothetical protein \\
\hline GGCCGTACAA & XM_641229.1 & 14 & acyl-CoA binding protein $(a c b A)$ \\
\hline GGGTATCACT & XM_633934.1 & 14 & hypothetical protein \\
\hline GGGTATCGCA & EĀL73180 & 14 & ADP/ATP translocase $($ ancA) \\
\hline GTGCAAATTG & XM_641171.1 & 14 & putative countin receptor $\mathrm{Cnr} 9(\mathrm{cnrl})$ \\
\hline GTTGACACTC & XM_633034.1 & 14 & 40S ribosomal protein $\mathrm{S} 15$ (rps 15$)$ \\
\hline TAAAAATATT & XM_631437.1 & 14 & $\begin{array}{l}\text { putative protein serine/threonine kinase (irlE)/LTR- } \\
\text { retrotransposon Skipper }\end{array}$ \\
\hline TCATTCACTA & XM_633173.1 & 14 & hypothetical protein \\
\hline TCCGGTGTCA & XM_638802.1 & 14 & catalase $($ catA $)$ \\
\hline TGAAATATAA & XM_640620.1 & 14 & 13/13 LISK family protein kinase \\
\hline TGATGGTGGT & XM_633438.1 & 14 & $\begin{array}{l}\text { hypothetical protein pleckstrin homology }(\mathrm{PH}) \\
\text { domain containing protein }(G x c Q) \text { mRNA }\end{array}$ \\
\hline TGATGTTGCC & $\begin{array}{l}\text { XM_631158.1, } \\
\text { U67940 }\end{array}$ & 14 & P14 domain-containing protein $(r c d G G)$ ORFveg 106 \\
\hline TGGACCTGGA & XM_631420.1 & 14 & cyclase associated protein (cap) \\
\hline TGGATAGATA & XM_633869.1 & 14 & proteosome subunit beta type 7 ( $p s m B 7)$ \\
\hline TGGTTCACCA & XM_633383.1 & 14 & mannose-6-phosphate isomerase (mpi)/countin \\
\hline TGTGGTCCAC & XM_638093.1 & 14 & hypothetical protein cytoplasmic dynein heavy chain $(d h c A)$ \\
\hline TTCAATGTAT & XM_001134613.1 & 14 & ubiquinol-cytochrome-c reductase hinge protein $(\operatorname{clec} 6 A)$ \\
\hline TTGCCAATGG & XM_641284.1 & 14 & $\mathrm{CD} 9(c d p 9)$ \\
\hline AAAATGGTAT & EAL71195 & 13 & 5'AMP activated gamma subunit (prkag) \\
\hline AACTAAATTA & AC116956.2 & 13 & chromosome 2 map \\
\hline AAGATACTGA & XM_633862.1 & 13 & phosphoprotein phosphatase $\mathrm{A}(p p p A)$ \\
\hline AATAATGAGT & XM_639860.2 & 13 & hypothetical protein putative polyketide synthesis ( $p k s 15)$ \\
\hline AATTACCTAC & XM_631966.1 & 13 & alpha tubulin $(t u b A)$ \\
\hline AATTTCAGAC & $\begin{array}{l}\text { AY953941.1, } \\
\text { AY953940.1 }\end{array}$ & 13 & U1B snRNA/U1A snRNA \\
\hline AGAGATTGGA & XM_629514.1 & 13 & malate dehydrogenase $(m d h B)$ \\
\hline ATCATAACCA & XM_641793.1 & 13 & hypothetical protein \\
\hline ATGACATCGG & V00192.1 & 13 & 5.8S ribosomal RNA \\
\hline ATGGATGAAT & XM_640496.1 & 13 & 13/13 RING Zn finger-containing protein ( $d g 1106)$ \\
\hline ATGGTTTCGG & XM_001134587.1 & 13 & 4-hydroxyphenylpyruvate dioxygenase \\
\hline CAAAAATATA & X⿳亠丷厂二_633368.1 & 13 & hypothetical protein \\
\hline CATATAATAT & AB007024.1 & 13 & DAPS-1 mRNA for proteosome subunit \\
\hline CGCATAATCA & XM_640523.2 & 13 & BEACH domain-containing protein $(l v s B)$ \\
\hline GAGAACTGCA & XM_638794.1 & 13 & pleckstrin homology $(\mathrm{PH})$ domain-containing protein \\
\hline GTAAACCACC & EĀL63602 & 13 & small aggregate formation $(\mathrm{smlA})$ \\
\hline GTAGAAAAAT & XM_637377.1 & 13 & valyl tRNA synthetase (valS1) \\
\hline GTAGAACCTC & XM_630153.1 & 13 & 13/13 hydroxyacylglutathione hydrolase ( $g l o B 2$ ) \\
\hline GTCATCCAAC & AF482388.1 & 13 & $\mathrm{ABC}$ transporter $\mathrm{AbcG} 9(a b c G 9)$ \\
\hline GTCGTGATAC & XM_639901.1 & 13 & seryl-tRNA synthetase (ser $S$ ) \\
\hline GTGAAAAGGA & XM_641077.1 & 13 & alpha-glucosidase II $(\bmod A)$ \\
\hline GTGTTGGTTC & XM_633060.1 & 13 & hypothetical protein \\
\hline GTTAGGTGAT & XM_630798.3 & 13 & FNIP repeat-containing protein DDB_0238003 \\
\hline GTTGTGTGTA & XM_637503.1 & 13 & hypothetical protein DDBdraft_0203368 \\
\hline GTTTCGTTTC & XM_630703.1 & 13 & hypothetical protein DDBdraft_0188832 \\
\hline TGAAAGATTA & XM_632907.1 & 13 & LIM domain-containing protein DDB_0233145 \\
\hline TTAATTGGTA & EĀL61182 & 13 & rab4 \\
\hline
\end{tabular}




\section{Ribosome-related genes and other RNAs}

Genes that produce components of the ribosome comprise $20 \%$ of the amoeba transcriptome (Table 2). This category includes the third most highly expressed gene, $40 \mathrm{~S}$ ribosomal protein S29, and 11 of the 28 genes expressed at the level of 100 copies or higher. Forty-nine different ribosomal proteins, initiation and elongation factors, several rRNA genes, and a potential regulator of RNA synthesis (NOT2/NOT3/NOT5 family protein) were also present at moderate to high expression levels. These data suggest that protein synthesis is a major function of amoebae.

Table 2. Ribosome-related genes and other RNA molecules of Dictyostelium discoideum.

\begin{tabular}{|c|c|c|c|}
\hline Dictyostelium tag sequence & ID & Count & Description \\
\hline TGCAGACGTT & XM_640038.1 & 308 & 40 S ribosomal protein S29 (rps29) \\
\hline CTGGTTACTC & XM_64088.1 & 168 & protein synthesis elongation factor 1-alpha (tef2) \\
\hline GAAAGACGTC & XM_15384.1 & 168 & V12 mRNA/ribosomal protein 1024 (rps9) \\
\hline GAAAAGAGAA & XM_635906.1 & 155 & $40 \mathrm{~S}$ ribosomal protein $\mathrm{S} 6(r p s 6)$ \\
\hline CTCGTCCTTA & XM_636364.1 & 154 & ribosomal protein L18 (rpl18) \\
\hline GATATTGAAG & XM_637875.1 & 151 & ribosomal protein L10a ( $r p l 10 \mathrm{a})$ \\
\hline TGTAGAGGTG & XM_637392.1 & 139 & 60 S ribosomal protein L4 (rpl4) \\
\hline CGTAAACCAG & X15383.1 & 122 & Ribosomal protein ( $r p 119)$ vegetative specific V14 gene \\
\hline GTGGTTTGAA & XM_639670.1 & 112 & $40 \mathrm{~S}$ ribosomal protein $\mathrm{S} 30($ rps 30$)$ \\
\hline GGGTAAGATT & XM_641702.1 & 110 & ribosomal protein L35a ( $r p l 35 \mathrm{a})$ \\
\hline GCCACTTTCT & XM_628965.1 & 100 & ribosomal protein s2 $(r p s 2) /$ LLRep3 \\
\hline TGCTATCCAC & XM_635481.1 & 95 & 40 S ribosomal protein S26 (rps 26$)$ \\
\hline GCCGCTCAAG & XM_630185.1 & 94 & 40S ribosomal protein $\mathrm{S} 14$ (rps 14$)$ \\
\hline TGGTTACAAG & XM_641274.1 & 94 & ribosomal protein L36a ( $r p l 36 \mathrm{a})$ \\
\hline AGAGGTCTCA & XM_636637.1 & 93 & vegetative specific gene V18 gene for ribosomal protein/ribosomal protein L11 ( $r p l 11)$ \\
\hline GTCGTCCAGG & XM_633034.1 & 92 & $40 \mathrm{~S}$ ribosomal protein $\mathrm{S} 15(r p s 15)$ \\
\hline TAACAACCGT & XM_641608.1 & 90 & $40 \mathrm{~S}$ ribosomal protein $\mathrm{SA}(r p s \mathrm{~A})$ \\
\hline GTCGTTCTTA & X00134.1 & 88 & 17S small subunit ribosomal RNA gene \\
\hline GTGTTAACGA & XM_639972.1 & 84 & ribosomal protein L15 (rpl15) \\
\hline GGTACCAAAG & XM_636854.1, XM_642089.1 & 82 & ribosomal protein L31 ( $r p l 31)$ /putative myb transcription factor \\
\hline CCACTACAAC & X15710.1, XM_639100.1 & 80 & ribosomal protein $\mathrm{L} 2 / 60 \mathrm{~S}$ ribosomal protein $\mathrm{L} 8(r p l 8)$ \\
\hline CGCTGGTGCA & XM_6317̄26.1 & 69 & ribosomal protein $\mathrm{L} 10 \mathrm{E}(r p l 10)$ \\
\hline ATCAAATCTG & XM_629345.1, U78756.1 & 68 & $40 \mathrm{~S}$ ribosomal protein $\mathrm{s} 3(r p s 3),(r p g G)$ \\
\hline GAAGTCGGTA & AY171066.1 & 64 & extrachromosomal palindromic ribosomal RNA \\
\hline GATGTCATCT & XM_639821.1 & 64 & 40 S ribosomal protein $\mathrm{S} 4$ (RPS4) \\
\hline CCTCTGGTAT & XM_640073.1 & 63 & 40 S ribosomal protein $\mathrm{S} 23$ (rps 23 ) \\
\hline GAGAAAACCA & XM_640259.1 & 63 & ribosomal protein L32 (rpl32) \\
\hline GATAAAAAAG & XM_637269.1 & 62 & $40 \mathrm{~S}$ ribosomal protein $\mathrm{S} 20(r p s 20)$ \\
\hline GTAAACCAAT & XM_638427.1 & 61 & AX4 RNA polymerase I, largest subunit ( $r p a 1$ ) \\
\hline GCTGCTCACT & XM_636632.1 & 57 & ribosomal protein L21 (rpl21) \\
\hline GTTGTAACGG & X00134.1 & 54 & 18S rRNA gene \\
\hline AATCACCCAA & XM_638028.1 & 52 & $40 \mathrm{~S}$ ribosomal protein $\mathrm{S} 18$ (rps 18$)$ \\
\hline GGCCACCGAA & XM_629870.1 & 52 & $40 \mathrm{~S}$ ribosomal protein $\mathrm{S} 8(r p s 8)$ \\
\hline TAAGATTGTT & X1-14970.1 & 52 & translation initiation factor $e I F-4 D$ \\
\hline TTCAACTGTT & XM_637485.1 & 51 & 40 S ribosomal protein S27 (rps27) \\
\hline TGCTACTATC & XM_642726.1 & 48 & 60 S ribosomal protein L9 $(r p l 9)$ \\
\hline CAGTAGCTAA & AY007805.1 & 44 & ribosomal protein $\mathrm{L} 36$ \\
\hline AATTCTTAAT & XM_629919.1, XM_629706.1 & 43 & ribosomal protein (L3) gene/AX4 aminomethyltransferase ( $r p l 3)$ \\
\hline AACTCTGGTC & XM_632836.1 & 41 & $40 \mathrm{~S}$ ribosomal protein $\mathrm{s} 5$ ( $r p s 5)$ \\
\hline CCAACCCGTT & XM_640574.1 & 39 & ribosomal protein L27 (rpl27) \\
\hline CAAAACCCAG & Ū78759.1 & 38 & IfdA translation initiation factor (ifdA) \\
\hline TCCGCCTTAG & XM_632431.1 & 37 & putative GATA-binding transcription factor (DDB_0216328) mRNA, complete cds \\
\hline AAAATAATAA & XM_636161.1 & 36 & YELA translation initiation factor $(y e l A)$ \\
\hline AAAGTTAGAG & XM_629874.1 & 36 & ribosomal protein L13 (rpl13) \\
\hline TACTTTACCC & XM_639670.1 & 34 & $40 \mathrm{~S}$ ribosomal protein $\mathrm{s} 30($ rps 30$)$ \\
\hline AGAGGTATCC & XM_631629.1 & 32 & elongation factor 2 \\
\hline TCCCCGTACA & XM_633420.1 & 32 & NOT2/NOT3/NOT5 family protein \\
\hline
\end{tabular}

Continued on next page 
Table 2. Continued.

\begin{tabular}{lccl}
\hline Dictyostelium tag sequence & ID & Count & Description \\
\hline TGTTCACCAT & XM_633215.1 & 32 & ribosomal protein L17 $(s p l 17)$ \\
GACCACAACG & XM_637999.1 & 31 & 40 S ribosomal protein s15 $(r p s 15 \mathrm{a}) / 40$ S ribosomal protein S24 \\
TGTTGCCCTC & XM_637474.1 & 28 & 60S ribosomal protein L7a $(r p l 7 \mathrm{a})$ \\
AAGAAAACTC & XM_638053.1 & 27 & ribosomal protein L7 $(r p l 7)$ \\
AGATTCAAAT & XM_631705.1 & 27 & ribosomal protiein L12 $(r p l 12)$ \\
CTTGGACTAA & XM_633462.1 & 26 & 40 S ribosomal protein $s 13(r p s 13)$ \\
TCTCAAGATC & AY007804.1 & 25 & ribosomal protein L28 $(r p l 28) 13 / 13$ \\
GCTACCGTCA & XM_630705.1 & 23 & ribosomal protein L23 $(r p l 23)$ \\
AATGCTTTCA & XM_632600.1 & 21 & 60S ribosomal acidic phosphoprotein P0 \\
GTGGTGGGAA & XM_640047.1 & 18 & putative small nuclear ribonucleoparticle-associated protein DDB_0233178 \\
AATGGATCAA & AY171066.1 & 16 & extrachromosomal palindromic ribosomal RNA \\
AAACCGTCAA & XM_633574.1 & 15 & 40 S ribosomal protein S12 $(r p s$ 12) \\
AAGAGTGGTA & XM_641660.1 & 15 & ribosomal protein L30 $(r p l 30)$ \\
ATTGAGAGAG & XM_642726.1 & 15 & 60S ribosomal protein L9 $(r p l 9)$ \\
CCGGATCACT & AY171065S2 & 15 & extrachromosomal palindromic ribosomal RNA \\
GTTGACACTC & XM_633034.1 & 14 & 40 S ribosomal protein S15 $(r p s 15)$ \\
AATTTCAGAC & AY953941.1, AY953940.1 & 13 & U1B snRNA/U1A snRNA \\
ATGACATCGG & V00192.1 & 13 & 5.8S ribosomal RNA \\
\hline
\end{tabular}

Table 2 includes the RNA polymerase I large subunit, expressed at moderate levels (61 copies), suggesting an active transcription rate. Table 2 also includes a few other types of RNA molecules such as extrachromosomal palindromic ribosomal RNA and the spliceosome components U1B/U1A snRNA, which are expressed at low levels.

\section{Enzymes and components of basic metabolism}

Numerous genes in the amoeba-stage transcriptome (18\%) produce the enzymes, regulatory molecules, modifying enzymes, and structural components of basic metabolism (Table 3 ). This group includes three of the 28 genes expressed at more than 100 copies, including the fourth-highest expressed gene in the transcriptome, a putative glycoside hydrolase.

The remainder of the genes in this group were expressed at moderate to low levels. Components of glycolysis, the tricarboxylic acid cycle, fatty acid oxidation, and the electron transport chain were found, as well as six different kinases and three different phosphatases. While the electron transport chain components reside in the mitochondria, we included them in the metabolism table for clarity.

Several biosynthetic enzymes were also expressed, including 13 different polyketide synthetases, and three t-RNA synthetases. While basic-metabolism proteins comprised a large group of expressed genes, the moderate level of expression suggests that these are basic maintenance functions of amoebae.

\section{Actin, actin-binding, and cytoskeleton-related genes}

With the exception of the hisactophilin II gene, the 26 actin-related genes included in Table 4 were expressed at moderate to low levels. They represent $8 \%$ of the transcriptome. These genes represent all of the major categories of cytoskeleton components, including actin-binding, capping, severing, cross-linking, and membrane associating, essential for maintaining amoeba shape, and allowing motility (Eichinger et al., 2005). Two myosin genes, mhcA and myoB, were included in this category due to their predicted role in chemotaxis (Postma et al., 2004). 
Table 3. Enzymes and components of basic metabolism in Dictyostelium discoideum.

\begin{tabular}{|c|c|c|c|}
\hline Dictyostelium tag sequence & ID & Count & Description \\
\hline CAAAGACTTT & XM_640564.1 & 297 & AX4 putative glycoside hydrolase/Mus musculus zinc finger protein 119 (Zfp 119$)$ \\
\hline TGAAATATAT & XM_001732911.1 & 104 & putative polyketide synthase $(p k s)$ \\
\hline GGGTGATAAT & XM_633636.1 & 103 & histidine kinase (dhkG) mRNA, complete cds \\
\hline TGCTGTCTCT & XM_633409.1 & 79 & glycyl-tRNA synthetase (glys) $(12 / 12)$ \\
\hline AAAAATCAAA & XM_639860.2 & 62 & putative polyketide synthase ( $p k s 15)$ \\
\hline GGTAATGGTA & XM_634924.1 & 59 & cyclophilin $(p p i A)$ \\
\hline GTATGATAAT & XM_638765.1 & 50 & glyceraldehyde-3-phosphate dehydrogenase ( $g p d A$ ) \\
\hline GTGAATCAAA & XM_635424.1 & 49 & putative alpha-amylase $(a m y A)$ \\
\hline AAAGAAGATG & XM_642543.1 & 45 & S-adenosyl-L-homocysteine hydrolase $(\operatorname{sah} A)$ \\
\hline CTGATAATGA & XM_642071.1, U23957.1 & 42 & acetylornithine deacetylase $(\arg E) / \mathrm{P} 52 \mathrm{D}$ \\
\hline TTGACATTGA & M91383.1 & 42 & thioredoxin $(\operatorname{tr} x 3)$ \\
\hline GAAAGATGGA & XM_639860 & 38 & putative polyketide synthase ( $p k s 15)$ \\
\hline AAAATTTACA & XM_001732912.1 & 36 & putative polyketide synthase \\
\hline TAGATCAGAA & XM_634139.1 & 32 & phosphopyruvate hydratase (enoA) \\
\hline TTTGGTTATG & XM_630291.1 & 30 & S-adenosylmethionine synthetase (met $K$ ) \\
\hline GGATGATGAA & XM_628865.1, U15926.1 & 29 & putative polyketide synthase/elongation factor $1 \mathrm{~b}(p k s 1)$ \\
\hline GGATGGTGGT & XM_638832.1 & 29 & acetyl-CoA C-acyltransferase \\
\hline GTCATTTCTT & XM_629407.1 & 29 & putative protein tyrosine phosphatase, dual specificity (DDB_0238566) mRNA, complete cds \\
\hline TGGATGTGAC & XM_001732949.1 & 29 & putative polyketide synthase $(p k s)$ \\
\hline ATATGACCCA & XM_632900.1 & 27 & type I phosphodiesterase nucleotide phosphatase family protein/PkiA (pkiA) \\
\hline GGCTCTCTCT & XM_638782.1 & 24 & fructose-bisphosphate adolase $(f b a)$ \\
\hline AAATCAAAAA & XM_001732910.1 & 23 & putative polyketide synthase \\
\hline TCATTCGTTT & XM_638104.1 & 23 & Glutamine amidotransferase (pyr 1-3) \\
\hline AAATCGCTTT & XM_636325.1 & 22 & nucleoside diphosphate kinase $(n d k M)$ \\
\hline GATTATCTTA & XM_632335.1 & 22 & polyketide synthase ( $p k s 25)$ \\
\hline CAGCACTAAA & M91382.1 & 22 & thioredoxin $(\operatorname{tr} x 2)$ \\
\hline CGCAAATGGT & XM_633239.1 & 21 & NADH dehydrogenase (ubiquinone) (DDB_0233208) \\
\hline GTGGTAGTAG & XM_642014.1 & 21 & diaminopimelate epimerase ( $d a f E$ ) \\
\hline GTTAGAGCCA & XM_641467.1 & 21 & succinate dehydrogenase (ubiquinone) \\
\hline TAAATTGGAC & XM_637030.1 & 21 & 6-phosphogluconate dehydrogenase ( $g n d$ ) \\
\hline AATTTGATGT & XM_639860.2 & 20 & putative poyketide synthase ( $p k s 15)$ \\
\hline TTTTCAATAA & XM_639838.2 & 20 & putative polyketide synthase ( $p k s 14)$ \\
\hline TAAAACAATT & XM_639838.2 & 18 & putative polyketide synthase ( $p k s 15)$ \\
\hline TGCTAACCTT & XM_632744.1 & 18 & alkyl-dihydroxyacetonephosphate synthase (agps) \\
\hline TTGCTTCTGA & XM_639860.2 & 18 & putative polyketide synthase ( $p k s 15)$ \\
\hline AAGATTAAAG & XM_641717.1 & 17 & putative protein serine/threonine kinase DDB_0230126 partial mRNA \\
\hline GGTGCCATCG & XM_638855.1 & 17 & amidophosphoribosyltransferase (purF) \\
\hline TGCAGTTTTA & XM_632827.2 & 17 & adenosine kinase $(a d k)$ \\
\hline GTTCACTCCT & XM_633237.1 & 16 & fatty acid desaturase $(\mathrm{fad} A)$ \\
\hline TCAATTCGAT & XM_633521.1 & 16 & ERG4/ERG24 ergosterol biosynthesis protein family protein (DDB_0232079) \\
\hline AAAACCATTG & XM_638265.1 & 15 & protein disulfide isomerase (dis) \\
\hline GAATTTTAAA & XM_639838.2 & 15 & hypothetical protein putative polyketide synthase ( $p k s 14)$ mRNA, complete cds \\
\hline CAAGTCGATA & XM_630022.1 & 14 & GlcNAc transferase (gnt2)/putative phosphatidylinositol 4-phosphate 5-kinase \\
\hline GATTGTCCAG & XM_635847.1 & 14 & ribonuclease $\mathrm{T} 2(d \mathrm{diA})$ \\
\hline GGCCGTACAA & XM_641229.1 & 14 & acyl-CoA binding protein $(a c b A)$ \\
\hline TAAAAATATT & XM_631437.1 & 14 & putative protein serine/threonine kinase ( $\mathrm{irlE}$ )/LTR-retrotransposon Skipper \\
\hline TCCGGTGTCA & XM_638802.1 & 14 & catalase $($ cat $A)$ \\
\hline TGAAATATAA & XM_640620.1 & 14 & 13/13 LISK family protein kinase \\
\hline TTCAATGTAT & XM_001134613.1 & 14 & ubiquinol-cytochrome-c reductase hinge protein $(\operatorname{clec} 6 A)$ \\
\hline AAGATACTGA & XM_633862.1 & 13 & phosphoprotein phosphatase $\mathrm{A}(p p p A)$ \\
\hline AATAATGAGT & XM_639860.2 & 13 & hypothetical protein putative polyketide synthesis ( $p k s 15)$ \\
\hline AGAGATTGGA & XM_629514.1 & 13 & malate dehydrogenase $(m d h B)$ \\
\hline ATGGTTTCGG & XM_001134587.1 & 13 & 4-hydroxyphenylpyruvate dioxygenase \\
\hline GTAGAAAAAT & XM_637377.1 & 13 & valyl tRNA synthetase ( valS1) \\
\hline GTAGAACCTC & XM_630153.1 & 13 & 13/13 hydroxyacylglutathione hydrolase ( $g l o B 2$ ) \\
\hline GTCGTGATAC & XM_639901.1 & 13 & seryl-tRNA synthetase (serS) \\
\hline GTGAAAAGGA & XM_641077.1 & 13 & alpha-glucosidase II $(\bmod A)$ \\
\hline
\end{tabular}




\begin{tabular}{|c|c|c|c|}
\hline Dictyostelium tag sequence & ID & Count & Description \\
\hline CTACTTTCGA & XM_635251.1, U13671.1 & 138 & actin binding protein $($ hat $B) /$ hisactophilin II $(h s I I)$ \\
\hline AAAATTAAAA & XM_641596.1 & 94 & $\operatorname{actin}(a c t 8)$ \\
\hline AAAATTAAAA & XM_640170.1 & 94 & actin $16($ act 16$)$ \\
\hline TTTAAGTGGT & XM_642699.1 & 76 & P17 protein \\
\hline GAAAAAGGTT & AF095929.1 & 59 & actin related protein $2(\operatorname{arp} B)$ \\
\hline ATGAAAGTGC & M25217.1 & 53 & $\operatorname{actin}(a c t)$ \\
\hline CTTGTGAGTT & XM_642699.1 & 51 & 17-kDa protein (p17) \\
\hline CAGTTGATCC & XM_641577.1 & 44 & gelation factor $(a b p c C)$ \\
\hline CGAATGGAAT & XM_642251.1 & 44 & actin binding protein (coronin) $(\operatorname{cor} A)$ \\
\hline TGTAACAACA & XM_632548.1 & 36 & putative actin binding protein \\
\hline GTGTGGTGAA & M92992.1, XM_628883.1 & 35 & cyclic AMP-regulated protein ( $\mathrm{p} 16) /$ actin binding protein \\
\hline CACACCAGTA & XM_634256.1 & 32 & $\mathrm{p} 34-\operatorname{Arc}(\operatorname{arp} E)$ \\
\hline TAAAAGCTCT & EAL66985 & 30 & $\mathrm{p} 41-\operatorname{Arc}(\operatorname{arpD})$ \\
\hline TTGAAACTCA & XM_640947.1 & 27 & actinobindin $(a b n B)$ \\
\hline AAATGTGATG & XM_641596.1, XM_640170.1 & 26 & $\operatorname{actin}(\operatorname{act} 8) / \operatorname{actin}(\operatorname{act} 16)$ \\
\hline AAGAAAGCTG & XM_632648.1 & 25 & myosin II heavy chain $(m h c A)$ \\
\hline CTCGCTCAAA & XM_636770.1 & 24 & 30,000 dalton actin bundling protein $(a b p B)$ \\
\hline CGAATTTATG & XM_640151.1 & 22 & actin capping protein CAP34 protein \\
\hline TGCTCTGTAG & XM_631290.1 & 21 & myosin IB (myoB) mRNA \\
\hline GGAGGTATGC & XM_641119.1 & 17 & WH2 domain-containing protein DDB_0231673 \\
\hline GTAAATCATT & XM_001134471.1 & 17 & annexin VII $(n x n A)$ \\
\hline CAACTCAATA & XM_001134515.1 & 16 & severin $(\operatorname{sev} A)$ \\
\hline CGATTCAATT & XM_632550.1 & 16 & putative actin binding protein \\
\hline CTTTCCGTGC & L46371 & 16 & cortexillin II \\
\hline ATTGCTTCTT & XM_634949.1 & 15 & putative actin binding protein (DDB_0232396) \\
\hline GTTGATGGGG & XM_641596.1, XM_640170.1 & 15 & $\operatorname{actin}(\operatorname{act} 8) / \operatorname{actin}(\operatorname{act} 16)$ \\
\hline ATGATCGTGG & XM_635251.1, U13671.1 & 14 & actin binding protein $($ hatB)/hisactophilin II ( $h s I I)$ \\
\hline TGGACCTGGA & XM_631420.1 & 14 & cyclase associated protein (cap) \\
\hline
\end{tabular}

\section{The endosomal system and membrane transport}

Components of the endosomal system, including receptor mediated endocytosis, lysosomal enzymes, trafficking and sorting molecules, protein folding systems, heat shock proteins, and several membrane transport systems, are described in Table 5. This group of genes represents $9 \%$ of the transcriptome, and includes three of the 28 genes expressed at more than 100 copies, two of which are lysosomal enzymes (cysteine proteinases CP4 and CP5). The remainder of the genes in this category were expressed at relatively low levels. Six of the genes are cysteine proteinases, reflecting the phagocytic nature of amoeba nutrition. One of the genes, cystatinA1, is an inhibitor of cysteine proteases. ADP-ribosylation factor 1 is included in this list because it is most likely to be associated with the Golgi apparatus and vesicle trafficking (Venkateswarlu et al., 2007; Weimer et al., 2008). The membrane transporters expressed in amoebae include two ABC transporters (abcG9 and abcG11) and one P-type ATPase that move ions and molecules across the plasma membrane, and components for one V-type ATPase (vatA and vatB), which probably maintains acidity in vacuoles. All of these transporters were expressed at low levels in the amoebae. 


\begin{tabular}{|c|c|c|c|}
\hline Dictyostelium tag sequence & ID & Count & Description \\
\hline GGGTATGGAT & XM_636628.2 & 195 & cysteine proteinase $(C P 4)$ \\
\hline TGGTTATCCA & $\mathrm{XM} 637389.2$ & 142 & D. discoideum $\mathrm{AX} 4$ putative transport protein (sec24l) mRNA, complete cds \\
\hline GGGTATCGAA & XM-639885.1 & 140 & cysteine proteinase CP5 ( cprE) \\
\hline AGTGTCGCTG & $\mathrm{XM}-631261.1$ & 39 & ADP-ribosylation factor 1 \\
\hline GGGTCTTGAC & $\mathrm{XM} 636633.1$ & 34 & cysteine proteinase $(c p r F) /$ cathepsin $\mathrm{O}$ ? \\
\hline GGAAATTTCT & $\mathrm{XM}^{-} 636553.1$ & 33 & cathepsin D $(c t s D)$ \\
\hline ACGAACTTTA & XM-633918.1 & 32 & calreticlin (crtA)/grp 94 mRNA for glucose-regulated protein 94 \\
\hline CAATTCGATA & XM_641945.1 & 29 & vacuolar protein sorting-associated protein (vps 26$)$ \\
\hline CAATGTTGAA & EĀL71934 & 26 & PepA \\
\hline GTTTCTAAAA & XM 641945.1 & 23 & vacuolar protein sorting-associated protein \\
\hline AAGATGATGA & $\mathrm{XM}^{-} 638093.1$ & 22 & heat shock protein \\
\hline GTGAAGATAA & $\mathrm{XM} 641945.1$ & 22 & vacuolar protein sorting-associated protein (DDB 0234203) mRNA, complete cds \\
\hline ATCATCAATA & $\mathrm{XM}-637516.1$ & 21 & vacuolar $\mathrm{H}+\mathrm{ATPase} \mathrm{B}$ subunit $($ vat $B)$ \\
\hline TATTGGTCAT & EĀL63838 & 21 & V-ATPase A subunit (vatA) \\
\hline GTAATATTGT & XM 640523.2 & 20 & BEACH domain containing protein (lvsB) \\
\hline TTATTGATTA & $\mathrm{XM}^{-} 641945.1$ & 20 & vacuolar protein sorting-associated protein (DDB 0234203) \\
\hline AAAAAGAAAT & EĀL68664 & 19 & clathrin light chain $(\operatorname{clc} A)$ \\
\hline GTACTGAATT & EAL64547 & 19 & crystal protein $(\operatorname{crvS})$ \\
\hline TAAAATTTTG & EAL71482 & 19 & rtoA \\
\hline CTCGTGTCTA & XM 629958.1 & 18 & cystatin A1 (cpiA) mRNA, complete cds \\
\hline GAGAACTTAT & $\mathrm{XM}-632346.1$ & 18 & SNF7 family protein ( $v p s 20)$ mRNA, complete cds \\
\hline AAGACACCAG & $\mathrm{XM} 639730.1$ & 17 & heat shock protein ( $h s p E-1)$ mRNA, complete cds \\
\hline AAGACACCAG & $\mathrm{XM}-639336.1$ & 17 & heat shock protein ( $h s p E-2)$ mRNA, complete cds \\
\hline TAACAAAGAA & $\mathrm{XM}^{-} 640523.2$ & 17 & hypothetical protein BEACH domain-containing protein $(l v s B)$ mRNA \\
\hline TGAAAAAAAA & $\mathrm{AF} \overline{4} 82390.1$ & 17 & transporter AbcG11 (abcG11) \\
\hline AAGCACAGGT & XM 630148.1 & 16 & hypothetical protein RING Zn finger-containing protein ( $v p s 8)$ mRNA, complete cds \\
\hline ATGATGAAGC & XM-629890.1 & 15 & vacuolar proton ATPase $100-\mathrm{kDa}$ subunit (vatM) \\
\hline GAAAATGGCC & $\mathrm{XM}-638890.1$ & 15 & hypothetical protein peptidase C19 family protein (DDB 0237738 ) mRNA \\
\hline TAAAAACAAC & $\mathrm{XM} 638317.1$ & 15 & $13 / 13$ natural resistance-associated macrophage protein ( \\
\hline TAAAGTTAGA & $\mathrm{XM}-632583.1$ & 15 & hypothetical protein vacuolar protein sorting-associated protein mRNA \\
\hline CGCATAATCA & $\mathrm{XM}-640523.2$ & 13 & BEACH domain-containing protein $($ lvs $B)$ \\
\hline GTCATCCAAC & AF̄ 82388.1 & 13 & $\mathrm{ABC}$ transporter AbcG9 $(a b c G 9)$ \\
\hline
\end{tabular}

\section{Small G-proteins}

Thirteen genes in the transcriptome (4\%) are G-proteins and their interacting partners (Table 6). The moderately high expression genes are small G-proteins that are most likely involved in motility via linkage with the actin cytoskeleton, such as Rac1A, and endosomal vesicle traffic, such as ADP-ribosylation factor, rab GTPase and rho GDP-dissociation inhibitor (Postma et al., 2004). The ras GTPase, which may be involved in transmembrane signal transduction, was expressed at relatively low levels.

\begin{tabular}{|c|c|c|c|}
\hline Dictyostelium tag sequence & ID & Count & Description \\
\hline GGATAACAAA & EAL69803 & 256 & $\mathrm{G}$ beta-like protein $(g p b B)$ \\
\hline AATTATTGAA & AF309947.1 & 43 & $\operatorname{Rac} 1 \mathrm{~A}(\operatorname{rac} 1 A)$ \\
\hline GTTGGGAAGA & AY0440851.1 & 39 & Rho GDP-dissociation inhibitor ( $r d i A)$ \\
\hline GCATTTGGAA & XM 631261.1 & 36 & ADP-ribosylation factor $(\operatorname{arf} A)$ \\
\hline ATGCTCCAAT & XM_628847.1 & 19 & leucine-rich repeat-containing protein $(\operatorname{lr} A)$ \\
\hline GGTTGGTAGT & $\mathrm{XM}^{-} 631716.1$ & 18 & leucine-rich repeat-containing protein ( $r r$ (roco4) \\
\hline GTCTCTCCAG & XM_632040.1 & 16 & RasGTPase-activating protein \\
\hline GTCACCACTA & XM_633438.1 & 15 & pleckstrin homology $(\mathrm{PH})$ domain-containing protein $(g x c Q)$ mRNA, complete cds \\
\hline AGATCATCTC & XM_642439.1 & 14 & rab GTPase $(r a b 6)$ \\
\hline GAGAAGAAGA & $\mathrm{XM}^{-} 638794.1$ & 14 & pleckstrin homology $(\mathrm{PH})$ domain-containing protein \\
\hline TGATGGTGGT & XM_633438.1 & 14 & hypothetical protein pleckstrin homology $(\mathrm{PH})$ domain containing protein $(G x c Q)$ mRNA \\
\hline GAGAACTGCA & XM 638794.1 & 13 & pleckstrin homology $(\mathrm{PH})$ domain-containing protein \\
\hline TTAATTGGTA & EĀL61182 & 13 & $r a b 4$ \\
\hline
\end{tabular}

Four pleckstrin homology (PH) domain genes, all expressed at low levels, are included in this category. Originally identified as proteins that bind to phosphatidylinositols, $\mathrm{PH}$ 
domains can also bind small G-proteins, such as Rho, Rac and ARF (Lemmon, 2004). Since the amoeba transcriptome does not contain any phosphatidylinositol metabolizing enzymes, it seems more likely that the $\mathrm{PH}$ domain proteins are associated with the small G-proteins in amoebae that are associated with cytoskeleton remodeling or vesicle traffic.

The most unusual member of this category is a G-beta-like protein, which is the sixth-mosthighly expressed gene in the transcriptome. No other potential heterotrimeric G-protein component is expressed in the transcriptome, so the function of this gene product in amoebae is difficult to ascertain.

\section{Ubiquitin and proteosome-related}

Five components of the proteosome and two ubiquitin ligases were expressed at moderate to low levels (Table 7). Ubiquitin was the second-highest expressed gene in the transcriptome. A single tag sequence is contained within five different predicted ubiquitin genes in Dictyostelium. We could not distinguish which ubiquitin gene(s) is (are) the source of this RNA. The wide range of processes that require ubiquitinated proteins explains the high level of expression.

\begin{tabular}{|c|c|c|c|}
\hline Dictyostelium tag sequence & ID & Count & Description \\
\hline ATTAGAGGAC & $\begin{array}{l}\text { XM_629433.1, } \\
\text { XM_629434.1, } \\
\text { XM_635973.1, } \\
\text { XM_635972.1, } \\
\text { XM_635971.1 }\end{array}$ & 440 & ubiquitin ( $u b q N, u b q O, u b q M, u b q L, u b q K)$ \\
\hline TAAGAGTTTT & XM_631880.1 & 35 & 26S proteosome regulatory subunit $\mathrm{S} 1$ ( $p s m D 1)$ \\
\hline CAAATGTAGT & $\mathrm{XM} 633133.1$ & 30 & RING Zn finger-containing protein (DDB-0232321) \\
\hline CTAATCGTCA & XM_637723.1 & 27 & ubiquitin $(u b q C)$ \\
\hline CATTCGTAAA & $\mathrm{XM}^{-} 640374.1$ & 23 & proteosomal alpha-subunit 7-1 (pntB) \\
\hline TATCTCAAGA & XM_629630.1 & 15 & $\begin{array}{l}26 \mathrm{~S} \text { proteosome subunit ATPase } 5(\text { psm } C 5) / \mathrm{HIV} 1 \\
\text { TAT-binding protein homologue }(t b p 1 \mathrm{O})\end{array}$ \\
\hline TGGATAGATA & XM_633869.1 & 14 & proteosome subunit beta type 7 ( $p s m B 7)$ \\
\hline ATGGATGAAT & $\mathrm{XM}^{-} 640496.1$ & 13 & 13/13 RING Zn finger-containing protein ( $\mathrm{dg} 1106)$ \\
\hline CATATAATAT & AB007024.1 & 13 & DAPS- 1 mRNA for proteosome subunit \\
\hline
\end{tabular}

\section{Tubulins and dyneines}

The 11 genes in this category produce the components of microtubules and molecular motors (Table 8). They participate in a variety of processes in amoebae, such as formation of the mitotic spindle, and provide structural support. All of these genes were expressed at moderate to low levels in the transcriptome.

\begin{tabular}{|c|c|c|c|}
\hline Dictyostelium tag sequence & ID & Count & Description \\
\hline TTTGTCAAGA & XM_631966.1 & 31 & alpha tubulin $(t u b a)$ \\
\hline AAGAACAAGC & $\mathrm{XM}-641070.1$ & 29 & beta tubulin gene $(t u b B)$ \\
\hline TAAACGAATC & XM_638093.1 & 25 & cytoplasmic dynein heavy chain $(d h c A)$ \\
\hline TCATTCGTTT & XM-638093.1 & 23 & cytoplasmic dynein heavy chain $(d h c A)$ \\
\hline GTCTTCAAGA & XM 639159.1 & 20 & colossin B mRNA ( $\operatorname{colB})$ \\
\hline TCAAATGCTG & $\mathrm{XM} 641218.1$ & 17 & major vault protein alpha ( $m v p$-alpha) \\
\hline TGGACTAAAT & $\mathrm{XM}-638093.1$ & 17 & hypothetical protein cytoplasmic dynein heavy chain $(d h c A)$ \\
\hline CAACCTCTGG & XM- 629574.1 & 16 & hypothetical protein colossin A (colA) mRNA, complete cds \\
\hline ATGCTGTCAT & XM 001134481.1 & 14 & hypothetical protein centrosomal protein CP224 (mtaA) mRNA, complete cds \\
\hline TGTGGTCCAC & X⿳亠丷⿵ 638093.1 & 14 & hypothetical protein cytoplasmic dynein heavy chain $(d h c A)$ \\
\hline AATTACCTAC & XM_631966.1 & 13 & alpha tubulin $(t u b A)$ \\
\hline
\end{tabular}




\section{DNA binding}

Three different histone genes were expressed at moderate to low levels in the amoebae (Table 9). The most striking gene in this category is a member of the CHD gene family, it had the fifth-highest level of expression in the transcriptome. This recently identified gene family activates or represses RNA transcription, depending on the component partners that are available (Hall and Georgel, 2007).

\begin{tabular}{lccl}
\multicolumn{2}{l}{ Table 9. DNA binding molecules of Dictyostelium discoideum. } \\
\hline Dictyostelium tag sequence & ID & Count & Description \\
\hline ATTTTTATTA & XM_633616.1 & 258 & $\begin{array}{l}\text { CHD gene family protein containing chromodomain, helicase domain, } \\
\text { and DNA-binding domain }\end{array}$ \\
CCAAAAGATA & XM_642485.1 & 59 & histone H3 $(d d H 3)$ \\
CCATCTTACA & XM_632592.1 & 34 & histone H2B domain containing protein $(H 2 B v 3)$ \\
GCTTTGGATG & XM_632592.1 & 22 & histone H2B domain containing protein $(H 2 B v 3)$ \\
ATGATGATGA & XM_633148.1 & 16 & nucleotide binding protein 1-like protein \\
\hline
\end{tabular}

\section{Cell adhesion}

The major cell adhesion molecule of amoebae, the cadA gene, was highly expressed, while the well-characterized contact site B gene was expressed at relatively low levels (Table 10). The two discoidin genes and the single extracellular matrix gene were originally predicted to be expressed only during later developmental stages. Their presence in the transcriptome indicates that they have a function in amoebae.

\begin{tabular}{lccl}
\multicolumn{4}{l}{ Table 10. Cell adhesion molecules of Dictyostelium discoideum. } \\
\hline Dictyostelium tag sequence & ID & Count & Description \\
\hline TTTGGTTGGT & XM_632956.1 & 175 & calcium-dependent cell adhesion molecule-1 $($ cadA $)$ \\
TGAAATAAAA & XM_637148.1 & 79 & extracellular matrix protein ST430 (13/13) \\
GGGTAATAAT & XM_629547.1 & 39 & discoidin II $(d s c E)$ \\
GAGTAAAGAT & XM_639850.1 & 24 & CsbA $(c s b A)$ and CsbB $(c s b B)$ \\
ATCAATGGGT & XM_639599.1 & 16 & discoidin-I D chain $(d s c D)$ \\
\hline
\end{tabular}

\section{Mitochondrium-related}

Several mitochondrial DNA sequences were detected by the SAGE method. Table 11 also includes nuclear encoded, mitochondria-specific proteins other than the electron transport chain components. The porin, superoxide dismutase, ADP/ATP translocase, and 5'AMP-activated gamma subunit were all expressed at relatively low levels.

\begin{tabular}{|c|c|c|c|}
\hline Dictyostelium tag sequence & ID & Count & Description \\
\hline CAAGTTGAAC & AB000109.1 & 133 & mitochondrial DNA \\
\hline AAAAATATAG & $\mathrm{AB} 000109.1$ & 44 & mitochondrial DNA \\
\hline ATGAGCAGTT & EAL63335 & 32 & mitochondrial DNA \\
\hline AGCTGGGTTT & EAL63335 & 28 & mitochondrial DNA \\
\hline GATAAACAAA & XM 640344.1 & 27 & porin $($ por $A)$ \\
\hline CTGATCAAGA & $\mathrm{XM}-642037.1$ & 21 & superoxide dismutase $(\operatorname{sod} A)$ \\
\hline GCTGTCGTCA & EĀL63335 & 21 & mitochondrial DNA \\
\hline GGGTATCGCA & EAL73180 & 14 & ADP/ATP translocase $(a n c A)$ \\
\hline AAAATGGTAT & EAL71195 & 13 & 5'AMP activated gamma subunit ( $p r k a g$ ) \\
\hline
\end{tabular}




\section{Quorum-sensing}

Six genes with possible quorum-sensing functions were expressed at moderate to low levels (Table 12). The small aggregate formation, countin and putative countin receptor are components of the countin factor complex, while conditioned medium factor and $r c d G G$ are two additional proposed quorum-sensing systems (Mahadeo and Parent, 2006).

\begin{tabular}{lccl}
\multicolumn{2}{l}{ Table 12. Quorum-sensing genes of Dictyostelium discoideum. } \\
\hline Dictyostelium tag sequence & ID & Count & Description \\
\hline GTGTAATCCA & EAL63602 & 77 & small aggregate formation $(\mathrm{smlA})$ \\
GTTATTGTAG & XM_638733.1 & 39 & conditioned medium factor $(\mathrm{cm} f A)$ \\
GTGCAAATTG & XM_641171.1 & 14 & putative countin receptor Cnr9 $(\mathrm{cnr})$ \\
TGATGTTGCC & XM_631158.1, U67940 & 14 & P14 domain-containing protein $(\mathrm{rcd} G G)$ ORFveg106 \\
TGGTTCACCA & XM_633383.1 & 14 & mannose-6-phosphate isomerase $(\mathrm{mpi}) / \mathrm{countin}$ \\
GTAAACCACC & EAL63602 & 13 & small aggregate formation $(\mathrm{sml} A)$ \\
\hline
\end{tabular}

\section{Hypothetical and genes of unknown function}

The largest group of genes in the transcriptome (76 tags) involves hypothetical proteins and genes with unknown functions (Table 13). We have defined hypothetical as those genes with recognized open reading frames in the Dictyostelium database. This group includes the most highly expressed gene in the transcriptome, dutA untranslated RNA, and four other genes expressed at greater than 100 copies. Several genes have structural motifs, such as four genes with EGF-like domains, but remain uncharacterized in any organism. Other genes have been identified in Dictyostelium, but their functions are unknown, such as the culmination-specific protein. Conversely, some genes have been identified in other organisms; but their function in Dictyostelium is unknown, such as the PhoPQ-activated pathogenicity-related protein.

Table 13. Hypothetical and genes of Dictyostelium discoideum of unknown function.

\begin{tabular}{lccl}
\hline Dictyostelium tag sequence & ID & Count & Description \\
\hline GGACTTAGAG & EAL64828 & 773 & dutA untranslated RNA \\
TCGCTTTAAT & XM_634269.1 & 154 & hypothetical protein \\
GCAATCAAAA & XM_630714.1 & 140 & hypothetical protein \\
GTATAGCTTA & EA_64828 & 130 & dutA RNA \\
CACTAATCAA & XM_635539.1 & 111 & hypothetical protein \\
GGGTGCCGAT & XM_630282.1 & 95 & hypothetical protein \\
AAATATAAAA & XM_635590.1 & 72 & hypothetical protein \\
GAGAATCATC & XM_632378.1 & 68 & hypothetical protein \\
GGTAATGGTA & XM_634924.1 & 59 & cyclophilin (ppiA) \\
TCCCTATTAA & XM_632008.1 & 59 & hypothetical protein \\
GGGTGCTGAC & XM_631551.1 & 57 & hypothetical protein \\
TGTCGATCCA & XM_641310.1 & 49 & hypothetical protein \\
TGGTGATGGT & XM_635474.1 & 46 & PhoPQ-activated pathogenicity-related protein (aprA) \\
GCAGGAAAAA & XM_636572.1 & 45 & hypothetical czl protein \\
TACTCTTTCT & AF203735.1 & 42 & culmination specific protein 45D (45D) \\
GAACCAGCCT & XM_638827.1 & 41 & hypothetical protein \\
GGATCCAGTG & XM_641936.1 & 41 & hypothetical protein DDB_0190094 partial mRNA \\
TCCATCATCA & XM_640805.1 & 37 & EGF-like domain \\
AGTTTCAGCC & XM_640335.2 & 34 & hypothetical protein \\
GGGTACATAT & XM_636202.1 & 33 & hypothetical protein \\
GGTATGATGT & XM_638942.1 & 33 & hypothetical protein \\
\hline
\end{tabular}

Continued on next page 
Table 13. Continued.

\begin{tabular}{|c|c|c|c|}
\hline Dictyostelium tag sequence & ID & Count & Description \\
\hline AAGTCATCGA & XM_630012.1 & 32 & hypothetical protein (29C) \\
\hline GCGAATGCAC & XM_642513.1 & 25 & hypothetical protein \\
\hline GAGATAATCT & XM_631114.1 & 24 & hypothetical protein \\
\hline GTTTGTTTGG & $\mathrm{XM} 640382.1$ & 24 & EGF-like domain containing protein $(\mathrm{canC})$ \\
\hline TAATAAACTC & $\mathrm{AC} 116986.1$ & 24 & chromosome 2 map \\
\hline GTTTTGGTGG & XM_640757.1 & 23 & hypothetical protein \\
\hline TAAATAGATC & XM_630528.1 & 23 & hypothetical protein \\
\hline GTCACTTCTT & XM_639759.1 & 22 & hypothetical protein \\
\hline GGATCTGCTG & XM_630177.1 & 21 & hypothetical protein \\
\hline GTCAAGCAAA & XM_632222.2 & 21 & hypothetical protein \\
\hline TAAAAAAAAA & $\mathrm{AC} 117072.3$ & 21 & chromosome 2 map \\
\hline TAGAAGAAGA & XM_642384.1 & 21 & non-transporter $\mathrm{ABC}$ protein $(a b c F 4)$ \\
\hline TGCTCTGTAG & XM 639875.1 & 21 & major facilitator superfamily protein DDB 0238776 \\
\hline TTGCAGAAAT & XM_630145.1 & 21 & hypothetical protein DDBdraft_0183916 \\
\hline CTAATGGGTC & $\mathrm{XM} 638179.1$ & 20 & hypothetical protein DDB 0232153 \\
\hline GCTAGAAATG & XM_632378.1 & 20 & hypothetical protein DDB̄aft_ 0187197 \\
\hline AAAAACACAT & XM_642213.1 & 19 & hypothetical protein DDBdraft_0216555 \\
\hline AAAAACACAT & XM_641971.1 & 19 & hypothetical protein DDBdraft_0216640 \\
\hline AAAAACACAT & XM_642177.1 & 19 & hypothetical protein DDBdraft_0216547 \\
\hline AGCAGAAGCA & XM_640867.2 & 19 & hypothetical protein DDBdraft_0215165 \\
\hline ATGCTCCAAT & XM_001733034.1 & 19 & hypothetical protein DDBdraft_0215064 \\
\hline GGAATTACCA & 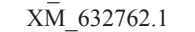 & 19 & hypothetical protein DDBdraft_ 0215883 \\
\hline TGGACCAAAG & XM_633814.1 & 19 & hypothetical protein DDB_0231746 \\
\hline CTGCTATTTC & XM_632737.1 & 18 & hypothetical protein DDBत̄raft_0186902 \\
\hline GTGATCTGTA & XM_637067.1 & 18 & hypothetical protein DDBdraft_0204455 \\
\hline GTGATCTGTA & XM_635945.1 & 18 & hypothetical protein DDBdraft_0204740 \\
\hline GTTGGTACTC & $\mathrm{XM} 631286.1$ & 18 & ankyrin repeat-containing protein DDB 0232969 \\
\hline TACCAAAATG & $\mathrm{XM}-640812.1$ & 18 & hypothetical protein DDBdraft $020169 \overline{6}$ \\
\hline TGCTAACCTT & XM_636773.1 & 18 & hypothetical protein DDBdraft_0205035 \\
\hline CACACCAACA & XM 638837.1 & 17 & hypothetical protein DDBdraft 0217482 \\
\hline GGAAACCTTT & XM_639002.1 & 17 & hypothetical protein DDBdraft_0217527 \\
\hline ACTAATCAAT & XM_638814.1 & 16 & hypothetical protein \\
\hline CGTAGAAAGC & XM_635431.1 & 16 & hypothetical protein \\
\hline GATGATGTAC & XM_636206.1 & 16 & hypothetical protein \\
\hline GTGAAAACAA & XM_635685.1 & 16 & hypothetical protein \\
\hline TGAAGATGGT & XM_633121.1 & 16 & hypothetical protein \\
\hline TGGAAATGGT & XM_637070.1 & 16 & EGF-like domain-containing protein \\
\hline ATAGAATGAG & $\mathrm{XM} 630305.1 /$ & 15 & penta EF hand calcium binding protein ( pefA)/apoptosis-linked gene $2(\operatorname{alg} 2 \mathrm{~A})$ \\
\hline ATGTTTGGTG & XM_639226.1 & 15 & hypothetical protein \\
\hline GGAAAATACA & XM 641146.2 & 15 & hypothetical protein \\
\hline GTGGTAGTTA & $\mathrm{A} \overline{\mathrm{C}} 114263$ & 15 & chromosome 2 map \\
\hline TATTTGATAC & XM_633831.1 & 15 & mMob1-like protein \\
\hline CTGGTCTTGT & XM_631976.1 & 14 & hypothetical protein \\
\hline GCCGTGATGA & XM_632857.2 & 14 & hypothetical protein \\
\hline GGGTATCACT & XM_633934.1 & 14 & hypothetical protein \\
\hline TCATTCACTA & $\mathrm{XM}^{-} 633173.1$ & 14 & hypothetical protein \\
\hline TTGCCAATGG & XM 641284.1 & 14 & CD9 $(c d p 9)$ \\
\hline AACTAAATTA & $\mathrm{AC} 116956.2$ & 13 & chromosome 2 map \\
\hline ATCATAACCA & XM_641793.1 & 13 & hypothetical protein \\
\hline CAAAAATATA & XM_633368.1 & 13 & hypothetical protein \\
\hline GTGTTGGTTC & XM_633060.1 & 13 & hypothetical protein \\
\hline GTTAGGTGAT & XM_630798.3 & 13 & FNIP repeat-containing protein DDB_0238003 \\
\hline GTTGTGTGTA & XM_637503.1 & 13 & hypothetical protein DDBdraft_0203368 \\
\hline GTTTCGTTTC & $\mathrm{XM} 630703.1$ & 13 & hypothetical protein DDBdraft 0188832 \\
\hline TGAAAGATTA & $\mathrm{XM} 632907.1$ & 13 & LIM domain-containing protein DDB 0233145 \\
\hline
\end{tabular}

\section{DISCUSSION}

The amoeba-stage transcriptome provides the information necessary to analyze the relative expression levels of all expressed genes, and make predictions about the functional capabilities of Dictyostelium amoebae. Our results, in some cases, confirm the results of 
previous studies that utilized molecular methods that depend on previously identified gene sequences, such as microarray analysis (Van Driessche et al., 2002; Urushihara et al., 2004). However, the value of an SAGE-produced transcriptome is that the entire array of expressed genes can be rank-ordered relative to each other, and identified based upon homology with the genome, rather than inclusion of specified sequences within a mixed probe. The result is a compilation of all gene products that are present within an amoeba. With this information, components of a process or pathway can be linked to this developmental stage, the functional capabilities identified, and their relative importance inferred.

Protein synthesis is a major function of amoebae, as previously reported (Van Driessche et al., 2002). Numerous ribosomal components are present and their expression levels vary from very high (300 copies) to relatively low (13 copies) levels. Conversely, the detectable proteosome components are few and not highly expressed (35 copies or less). Together these data suggest that the expressed genes are relatively stable, because the expression levels of the protein degradation machinery are about 10 times less than those of the protein synthesis machinery.

The moderate to high expression levels of the endosomal system components, transporters and lysosomal enzymes are indicative of the phagocytic/pinocytic nature of amoeba nutrition. The many metabolic enzymes expressed indicate that amoebae are utilizing nutrients for energy production and synthesis of necessary structural components. However, the relatively low expression levels of metabolic enzymes, particularly those of glucose oxidation and oxidative phosphorylation, suggest that this level is simply representative of basal metabolic rates for amoebae. Given the large amount of ATP needed for early development processes (aggregation), it appears that amoebae are not producing and storing ATP for later use.

The only identifiable transmembrane receptor expressed in the amoebae was the countin receptor, which, along with its cognate ligand countin was expressed at low levels (14 copies). These two gene products are components of a quorum-sensing system that also includes the small A gene product (Mahadeo and Parent, 2006). The conditioned medium factor (CMF) represents a second quorum-sensing system and was expressed at moderate to low levels, although its receptor CMFR1 is not present in the transcriptome (Clarke and Gomer, 1995). Other potential cell-signaling components include regulators of monomeric G-protein function, such as Rac1A or Rab6. However, in the absence of ras or receptor tyrosine kinases, these gene products are more likely involved in linking the cytoskeleton to the membrane or in vesicle trafficking. Therefore, quorum sensing appears to be the only form of transmembrane signaling that occurs in amoebae. These data support the paradigm that quorum-sensing systems activate the cAMP synthesis and detection systems required for aggregation, the earliest stage of multicellular development (Van Haastert et al., 1996). Furthermore, the absence of cAMP receptors or heterotrimeric G-protein $\alpha$-subunits indicates that amoebae at a density of $2.4 \times 10^{7}$ cells $/ \mathrm{mL}$ (the concentration used for this study) have not initiated development. While an amoeba culture of this density is likely approaching the stationary phase, the gene expression patterns in these amoebae demonstrate that they have not made the transition to multicellular development (Franck et al., 2008).

One of the surprising findings of the Dictyostelium genome sequence and analysis is the large number (43) of polyketide synthase genes present in the genome (Eichinger et al., 2005). The amoeba transcriptome includes 13 different polyketide synthases with a wide range of expression levels from high (104 copies) to low (13 copies). To date, the only clear function for polyketide synthase is in the biosynthetic pathway for differentiation-inducing factor-1 (Dif-1) (Kay, 1998; Ghosh et al., 2008). However, Dif-1 functions much later in 
development to induce the differentiation of stalk cells. Therefore, the products of the 13 amoeba-stage polyketide synthases are unknown.

The function of the most highly expressed gene in amoebae, dutA untranslated RNA (773 copies), is unknown (Yoshida et al., 1994; Hinas and Soderborn, 2007). Obviously, a gene expressed at a level almost twice that of the next highest gene has an important function, and identifying that function should produce important insights into what defines an amoeba. Non-coding RNAs, like $d u t$ A, function in a variety of ways in other organisms, such as chromatin remodeling or transcriptional or translational inhibition (Hinas and Soderbom, 2007). A recent report suggests that $d u t \mathrm{~A}$ untranslated RNA can function as a multicopy suppressor of STATa, a gene required for the last stage of development, culmination (Shimada and Kawata, 2007). A similar situation exists for the fifth-most-highly expressed gene in the amoeba transcriptome, chromodomain/helicase/DNA-binding domain or CHD protein (258 copies) (Hall and Georgel, 2007). CHD proteins in other organisms can function to increase or decrease transcription, depending on the available partner proteins. Possibly, amoebae remain undifferentiated because development genes are repressed at the transcription level by CHD and at the translation level by dutA. Understanding the mechanisms by which both of these gene products function should produce fruitful insights into developmental control of gene expression.

The large number of hypothetical gene products or genes of unknown function (76 genes), particularly those expressed at high levels ( $>100$ copies), indicates that there is much we do not know about the genes necessary to be an amoeba. The amoeba transcriptome provides a basis for selective characterization of genes that can fill the knowledge gaps, and enhance our understanding of the transition point from single cells to multicellular development.

\section{ACKNOWLEDGMENTS}

Research supported by NIH Grant \#P20 RR16454 from the BRIN/INBRE Program of the National Center for Research Resources, and an NSF EPSCoR Ph.D. fellowship to T.J. Whitney.

\section{REFERENCES}

Chisholm RL, Gaudet P, Just EM, Pilcher KE, et al. (2006). dictyBase, the model organism database for Dictyostelium discoideum. Nucleic Acids Res. 34: D423-D427.

Chung CY and Firtel RA (2000). Dictyostelium: A Model Experimental System for Elucidating the Pathways and Mechanisms Controlling Chemotaxis. In: Principles of Molecular Regulation (Conn PM and Means AR, eds.). Springer, New York, 99-114.

Clarke M and Gomer RH (1995). PSF and CMF, autocrine factors that regulate gene expression during growth and early development of Dictyostelium. Experientia 51: 1124-1134.

Eichinger L, Pachebat JA, Glockner G, Rajandream MA, et al. (2005). The genome of the social amoeba Dictyostelium discoideum. Nature 435: 43-57.

Firtel RA (1996). Interacting signaling pathways controlling multicellular development in Dictyostelium. Curr. Opin. Genet. Dev. 6: 545-554.

Franck C, Ip W, Bae A, Franck N, et al. (2008). Contact-mediated cell-assisted cell proliferation in a model eukaryotic single-cell organism: an explanation for the lag phase in shaken cell culture. Phys. Rev. E Stat. Nonlin. Soft Matter Phys. 77: 041905.

Ghosh R, Chhabra A, Phatale PA, Samrat SK, et al. (2008). Dissecting the functional role of polyketide synthases in Dictyostelium discoideum: biosynthesis of the differentiation regulating factor 4-methyl-5-pentylbenzene-1,3-diol. J. Biol. Chem. 283: 11348-11354.

Hall JA and Georgel PT (2007). CHD proteins: a diverse family with strong ties. Biochem. Cell Biol. 85: 463-476.

Hinas A and Soderbom F (2007). Treasure hunt in an amoeba: non-coding RNAs in Dictyostelium discoideum. Curr. Genet. 51: 141-159. 
Kay RR (1998). The biosynthesis of differentiation-inducing factor, a chlorinated signal molecule regulating Dictyostelium development. J. Biol. Chem. 273: 2669-2675.

Kenzelmann M and Muhlemann K (1999). Substantially enhanced cloning efficiency of SAGE (Serial Analysis of Gene Expression) by adding a heating step to the original protocol. Nucleic Acids Res. 27: 917-918.

Lemmon MA (2004). Pleckstrin homology domains: not just for phosphoinositides. Biochem. Soc. Trans. 32: 707-711.

Mahadeo DC and Parent CA (2006). Signal relay during the life cycle of Dictyostelium. Curr. Top. Dev. Biol. 73: 115-140.

Parent CA and Devreotes PN (1996). Molecular genetics of signal transduction in Dictyostelium. Annu. Rev. Biochem. 65: 411-440.

Postma M, Bosgraaf L, Loovers HM and Van Haastert PJ (2004). Chemotaxis: signalling modules join hands at front and tail. EMBO Rep. 5: 35-40.

Shimada N and Kawata T (2007). Evidence that noncoding RNA dutA is a multicopy suppressor of Dictyostelium discoideum STAT protein Dd-STATa. Eukaryot. Cell 6: 1030-1040.

Urushihara H, Morio T, Saito T, Kohara Y, et al. (2004). Analyses of cDNAs from growth and slug stages of Dictyostelium discoideum. Nucleic Acids Res. 32: 1647-1653.

Van Driessche N, Shaw C, Katoh M, Morio T, et al. (2002). A transcriptional profile of multicellular development in Dictyostelium discoideum. Development 129: 1543-1552.

Van Haastert PJ, Bishop JD and Gomer RH (1996). The cell density factor CMF regulates the chemoattractant receptor cAR1 in Dictyostelium. J. Cell Biol. 134: 1543-1549.

Velculescu VE, Zhang L, Vogelstein B and Kinzler KW (1995). Serial analysis of gene expression. Science 270: 484-487.

Venkateswarlu K, Brandom KG and Yun H (2007). PI-3-kinase-dependent membrane recruitment of centaurin- $\alpha 2$ is essential for its effect on ARF6-mediated actin cytoskeleton reorganisation. J. Cell Sci. 120: 792-801.

Verkerke-van Wijk I, Fukuzawa M, Devreotes PN and Schaap P (2001). Adenylyl cyclase A expression is tip-specific in Dictyostelium slugs and directs StatA nuclear translocation and CudA gene expression. Dev. Biol. 234: 151-160.

Weimer C, Beck R, Eckert P, Reckmann I, et al. (2008). Differential roles of ArfGAP1, ArfGAP2, and ArfGAP3 in COPI trafficking. J. Cell Biol. 183: 725-735.

Yoshida H, Kumimoto H and Okamoto K (1994). dutA RNA functions as an untranslatable RNA in the development of Dictyostelium discoideum. Nucleic Acids Res. 22: 41-46. 